\title{
MAXIMALLY STAR-FORMING GALACTIC DISKS. I. STARBURST REGULATION VIA FEEDBACK-DRIVEN TURBULENCE
}

\author{
Eve C. OstrikeR ${ }^{1}$ AND RAhUl SHetTy ${ }^{2}$ \\ ${ }^{1}$ Department of Astronomy, University of Maryland, College Park, MD 20742, USA; ostriker@astro.umd.edu \\ ${ }^{2}$ Zentrum für Astronomie der Universität Heidelberg, Institut für Theoretische Astrophysik, Albert-Ueberle-Str. 2, 69120 Heidelberg, Germany; \\ rshetty@ita.uni-heidelberg.de \\ Received 2010 November 28; accepted 2011 February 4; published 2011 March 22
}

\begin{abstract}
Star formation rates in the centers of disk galaxies often vastly exceed those at larger radii, whether measured by the surface density of star formation $\Sigma_{\mathrm{SFR}}$, by the star formation rate per unit gas mass, $\Sigma_{\mathrm{SFR}} / \Sigma$, or even by total output. In this paper, we investigate the idea that central starbursts are self-regulated systems in which the momentum flux injected to the interstellar medium (ISM) by star formation balances the gravitational force confining the ISM gas in the disk. For most starbursts, supernovae are the largest contributor to the momentum flux, and turbulence provides the main pressure support for the predominantly molecular ISM. If the momentum feedback per stellar mass formed is $p_{*} / m_{*} \sim 3000 \mathrm{~km} \mathrm{~s}^{-1}$, the predicted star formation rate is $\Sigma_{\mathrm{SFR}} \sim 2 \pi G \Sigma^{2} m_{*} / p_{*} \sim 0.1 M_{\odot} \mathrm{kpc}^{-2} \mathrm{yr}^{-1}\left(\Sigma / 100 M_{\odot} \mathrm{pc}^{-2}\right)^{2}$ in regions where gas dominates the vertical gravity. We compare this prediction with numerical simulations of vertically resolved disks that model star formation including feedback, finding good agreement for gas surface densities in the range $\Sigma \sim 10^{2}-10^{3} M_{\odot} \mathrm{pc}^{-2}$. We also compare to a compilation of star formation rates and gas contents from local and high-redshift galaxies (both mergers and normal galaxies), finding good agreement provided that the conversion factor $X_{\mathrm{CO}}$ from integrated $\mathrm{CO}$ emission to $\mathrm{H}_{2}$ surface density decreases modestly as $\Sigma$ and $\Sigma_{\text {SFR }}$ increase. Star formation rates in dense, turbulent gas are also expected to depend on the gravitational free-fall time at the corresponding mean ISM density $\rho_{0}$; if the star formation efficiency per free-fall time is $\varepsilon_{\mathrm{ff}}\left(\rho_{0}\right) \sim 0.01$, the turbulent velocity dispersion driven by feedback is expected to be $v_{z}=0.4 \varepsilon_{\mathrm{fff}}\left(\rho_{0}\right) p_{*} / m_{*} \sim 10 \mathrm{~km} \mathrm{~s}^{-1}$, relatively independent of $\Sigma$ or $\Sigma_{\mathrm{SFR}}$. Turbulenceregulated starbursts (controlled by kinetic momentum feedback) are part of the larger scheme of self-regulation; primarily atomic low- $\Sigma$ outer disks may have star formation regulated by ultraviolet heating feedback, whereas regions at extremely high $\Sigma$ may be regulated by feedback of stellar radiation that is reprocessed into trapped infrared.
\end{abstract}

Key words: galaxies: ISM - galaxies: kinematics and dynamics - galaxies: starburst - galaxies: star formation ISM: structure - turbulence

Online-only material: color figures

\section{INTRODUCTION}

Averaged over large scales in disk galaxies, the relationship between the mean surface density of star formation, $\Sigma_{\mathrm{SFR}}$, and the mean gaseous surface density, $\Sigma$, is observed to be superlinear, both in the local universe and at higher redshift (e.g., Kennicutt 1998b; Genzel et al. 2010a). This behavior reflects the increased efficiency (or shorter timescale) of star formation under conditions of higher mean gas density, which is correlated with higher $\Sigma$. Starburst regions within the central $\sim \mathrm{kpc}$ of galaxies, commonly observed as dust-enshrouded LIRGs and ULIRGs in which the interstellar medium (ISM) is predominantly molecular (Sanders \& Mirabel 1996; Genzel et al. 1998; Kennicutt 1998a; Solomon \& Vanden Bout 2005), represent the extreme of this behavior, with $\Sigma \sim 10^{2}-10^{4} M_{\odot} \mathrm{pc}^{-2}$ and the star formation (or gas conversion) timescale $t_{\mathrm{SF}, \mathrm{gas}} \equiv \Sigma / \Sigma_{\mathrm{SFR}}$ a factor of 10 or more below $t_{\mathrm{SF} \text {,gas }}$ in lower- $\Sigma$ regions of galaxies. Although earlier observations based on global averages suggested a simple power-law relationship between $\Sigma$ and $\Sigma_{\text {SFR }}$, these global relations have considerable scatter about the mean. More recently, evidence has emerged that the star formation regime within galactic centers at very high $\Sigma \gtrsim 100 M_{\odot} \mathrm{pc}^{-2}$ is distinct from the star formation regime that prevails in the main disks of galaxies at lower $\Sigma \lesssim 100 M_{\odot} \mathrm{pc}^{-2}$. Within the main disk, another change in the star formation regime appears to occur from "mid-disk" to "outer-disk." Typ- ically, both galactic center and mid-disk regions are primarily molecular, whereas outer disks are primarily atomic. Figure 1 provides a schematic, dividing the disk into different starforming regimes.

In main-disk regions where $\Sigma \lesssim 100 M_{\odot} \mathrm{pc}^{-2}$, highresolution observations have found that $\Sigma_{\mathrm{SFR}}$ is proportional to $\Sigma_{\text {mol }}$, the mean surface density of molecular gas, with a timescale $t_{\mathrm{SF}, \mathrm{mol}} \equiv \Sigma_{\mathrm{mol}} / \Sigma_{\mathrm{SFR}} \approx 2 \mathrm{Gyr}$ (Bigiel et al. 2008; Blanc et al. 2009). The value $\Sigma \sim 100 M_{\odot} \mathrm{pc}^{-2}$ that defines the upper limit for "main disks" is characteristic of the surface density $\Sigma_{\mathrm{GMC}}$ of individual giant molecular clouds (GMCs), as observed in the Milky Way and Local Group (Blitz et al. 2007; Sheth et al. 2008; Bolatto et al. 2008). The relation $\Sigma_{\mathrm{SFR}} \propto \Sigma_{\mathrm{mol}}$ for $\Sigma \lesssim \Sigma_{\mathrm{GMC}}$ is consistent with the idea that spatially isolated GMCs have an approximately constant specific star formation rate; this rate, and the value of $\Sigma_{\mathrm{GMC}}$, may be controlled by internal feedback processes within GMCs (e.g., Krumholz et al. 2006), although this is not yet completely understood. Over a typical GMC lifetime of $\sim 20 \mathrm{Myr}$ (Blitz et al. 2007), $\sim 1 \%$ of the gas must be converted to stars in order to yield $t_{\mathrm{SF} \text {,mol }} \approx 2 \mathrm{Gyr}$ within maindisk regions. Although there are uncertainties arising from limited resolution and calibration of the $\mathrm{CO}-$ to- $\mathrm{H}_{2}$ conversion (see Section 4), the relationship between $\Sigma_{\text {mol }}$ and $\Sigma_{\mathrm{SFR}}$ in galactic center regions with $\Sigma \gtrsim 100 M_{\odot} \mathrm{pc}^{-2}$ appears considerably steeper than linear. Presumably, this is because the molecular gas in galactic center regions with $\Sigma \gtrsim 100 M_{\odot} \mathrm{pc}^{-2}$ is 


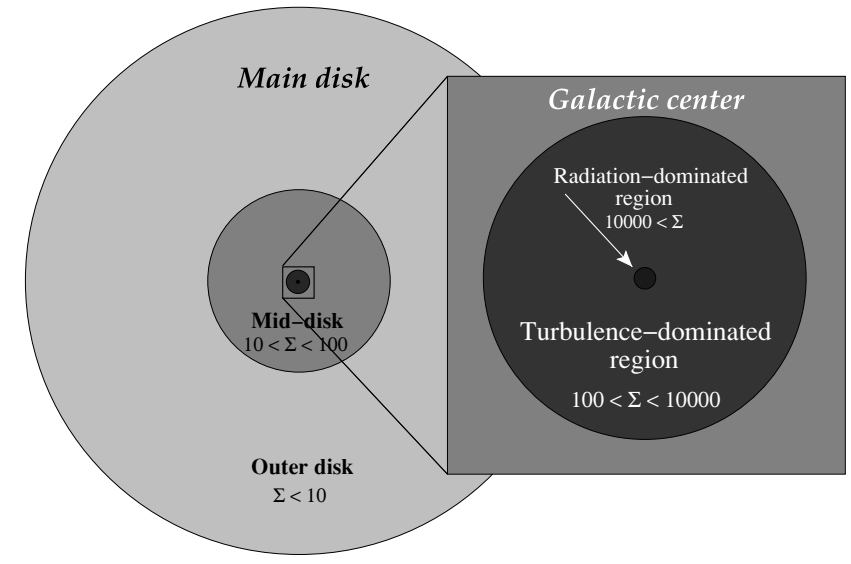

Figure 1. Schematic indicating the geography of galactic disk star formation; ranges for gaseous surface density $\Sigma$ (in $M_{\odot} \mathrm{pc}^{-2}$ ) in the various regions are approximate. Regulation of star formation in the main disk has recently been discussed by Ostriker et al. (2010). In this paper, we analyze self-regulation of star formation in the turbulence-dominated region within the galactic center for starburst disks.

at higher mean (volume) density than the molecular gas in main-disk GMCs having "standard" surface density $\Sigma_{\mathrm{GMC}} \sim$ $100 M_{\odot} \mathrm{pc}^{-2}$.

Molecular gas dominates mid-disk regions, but is a small fraction of the total in outer disks, where $\Sigma$ and the disk's stellar density $\rho_{*}$ are lower (Wong \& Blitz 2002; Blitz \& Rosolowsky 2004, 2006; Leroy et al. 2008). Ostriker et al. (2010) have recently advanced a theory to explain the starforming behavior and balance of phases in the main regions of disks at $\Sigma \lesssim 100 M_{\odot} \mathrm{pc}^{-2}$, based on the requirement that the diffuse $\mathrm{H}$ I gas must be in both thermal and dynamical equilibrium. In this theory, star-forming gravitationally bound clouds are assumed to have internal pressures much larger than their surroundings, consistent with local GMC observations. Thus, they are effectively isolated, and over their lifetimes are assumed to maintain a mean internal specific star formation rate consistent with the empirical main-disk value $t_{\mathrm{SF} \text {,mol }} \approx 2 \mathrm{Gyr}$. In equilibrium, individual gravitationally bound clouds form and disperse at equal rates, with neutral ISM gas cycling between the diffuse and gravitationally bound components. In outer-disk regions (typically $\Sigma \lesssim 10 M_{\odot} \mathrm{pc}^{-2}$, although this varies with $\rho_{*}$ ), the equilibrium fraction of gas in starforming bound clouds is just enough that the heating provided by the resulting stellar ultraviolet (UV) balances the cooling of the surrounding diffuse $\mathrm{H}$ I; here, $\Sigma_{\mathrm{SFR}} \propto \Sigma \sqrt{\rho_{*}}$. In middisk regions (typically $10 M_{\odot} \mathrm{pc}^{-2} \lesssim \Sigma \lesssim 100 M_{\odot} \mathrm{pc}^{-2}$ ), the ISM becomes predominantly molecular (by mass) because the heating provided by star formation would be insufficient to balance cooling in high-density warm atomic gas (which is compressed by the vertical disk gravity); here, $\Sigma_{\text {SFR }} \propto \Sigma_{\text {mol }} \propto \Sigma$. The model of Ostriker et al. (2010) assumes that most of the volume is filled by non-star-forming atomic gas, and thus is inapplicable to the starburst regime in galactic centers where $\Sigma \gtrsim 100 M_{\odot} \mathrm{pc}^{-2}$ and gas is mostly molecular.

Where $\Sigma$ is very high, the conditions throughout the ISM resemble those in the interior of locally observed GMCs, but are even more extreme in terms of the mean densities, the abundance of very high density gas, and the ratio of the turbulent velocity dispersion $v_{\text {turb }}$ to the thermal speed $v_{\text {th }}$ (e.g., Scoville et al. 1991; Downes et al. 1993; Downes \& Solomon 1998; Gao \& Solomon 2004). Supersonic turbulence is known to be a dominant process controlling star formation regulation within GMCs (Mac Low \& Klessen 2004; McKee \& Ostriker 2007), so it is expected to be important in starburst regions as well. Main-disk GMCs are relatively isolated entities, in the sense that their internal dynamical timescales are short compared to the orbital, epicyclic, and vertical oscillation times in the galactic potential $\left(\Omega^{-1}, \kappa^{-1}\right.$, and $v^{-1}$, respectively). In galactic center regions, however, the gravitational potential is deeper, such that $\Omega, \kappa$, and $v$ are all substantially higher, with timescales $\lesssim 10$ Myr. Thus, the galactic environment may also significantly impact the development of star formation.

Turbulence in the ISM both inhibits and encourages star formation. For regions large compared to the energy (and momentum) injection scale, turbulence provides an effective pressure that opposes gravity. In the ISM, this helps to regulate the mean density averaged over the disk thickness $\bar{\rho}$, and therefore the large-scale self-gravitational timescale $\left\langle t_{\text {grav }}\right\rangle \propto \bar{\rho}^{-1 / 2} \propto$ $(H / \Sigma)^{1 / 2}$. Here, the gas disk semi-thickness $H$ varies with turbulent velocity dispersion as $H \propto \sigma_{z}$ or $\sigma_{z}^{2}$ depending on whether stellar or gas gravity dominates the vertical potential gradients. In either case, larger turbulent velocities raise $H$ and $\left\langle t_{\text {grav }}\right\rangle$ for the ISM disk, while larger stellar and gas densities lower $H$ and $\left\langle t_{\text {grav }}\right\rangle$. On scales smaller than the turbulent injection, supersonic compression creates local overdense regions $\rho_{\text {local }} \gg \bar{\rho}$ that potentially can collapse more rapidly than the larger scale regions containing them since $t_{\text {grav, local }} /\left\langle t_{\text {grav }}\right\rangle \propto\left(\rho_{\text {local }} / \bar{\rho}\right)^{-1 / 2} \ll 1$. However, turbulent rarefactions and shear also destroy overdensities. Because $t_{\text {grav,local }}$ depends on density, turbulence will disperse the moderate-density structures before self-gravity can concentrate them further, while allowing the highest-density regions (amounting to a small fraction of the mass) to collapse and form stars. Theoretical arguments suggest that the fraction of mass in turbulent systems that collapses to form stars will be relatively insensitive to the velocity dispersion (Krumholz \& McKee 2005; see also Section 5), so that the overall star formation rate is primarily governed by the gravitational time on large scales, $\left\langle t_{\text {grav }}\right\rangle$. Since massive stars energize the ISM, raising the velocity dispersion and $\left\langle t_{\text {grav }}\right\rangle$, the associated feedback loop may allow star formation rates to be self-regulated.

In this paper, we investigate the process of self-regulation via turbulent driving, for application to understanding what controls star formation rates in starburst disks. For molecule-dominated regions, cooling times are short, so it is the injection of momentum, rather than energy, that is essential in defining the dynamical state of the disk. Thus, we begin in Section 2 by considering the implications of maintaining force balance in the vertical direction, which imposes a requirement on the input momentum flux associated with star formation feedback. We show that for disks where supernovae dominate the feedback, the star formation rate is expected to vary as $\Sigma_{\mathrm{SFR}} \propto \Sigma^{2}$. At very high surface density in optically thick disks, radiation pressure can exceed the turbulent pressure driven by supernovae, which leads to $\Sigma_{\mathrm{SFR}} \propto \Sigma$. In Section 3, we compare our prediction for $\Sigma_{\mathrm{SFR}}$ versus $\Sigma$ in turbulence-dominated disks to the results of numerical simulations, for which feedback is explicitly implemented in local vertically resolved models of self-gravitating, rotating disks. Section 4 compares the prediction for $\Sigma_{\text {SFR }}$ versus $\Sigma$ to observations of galaxies in the regime where the turbulencedominated model is expected to apply. Both the numerical simulations and the observations are consistent with the simple analytic model. In Section 5, we connect to models relating star formation rates to $\left\langle t_{\text {grav }}\right\rangle$, and discuss how the mean internal properties (including velocity dispersion and disk thickness) of turbulence-dominated, feedback-regulated disks are expected 
to depend on $\Sigma$ and the parameters that characterize the star formation feedback. We conclude in Section 6 with a brief summary and discussion.

\section{A SIMPLE MODEL FOR MOMENTUM-CONTROLLED, SELF-REGULATED DISK STAR FORMATION}

Consider a region of a galactic disk with gas surface density $\Sigma$, residing within a bulge of uniform density $\rho_{b}$, such that the gasdisk gravitational potential is $\Phi_{g}$ and the bulge potential is $\Phi_{b}$. We assume that the gas disk's internal structure is similar to that of a GMC, in that it is highly inhomogeneous (due to supersonic turbulence) but most of the gas concentrations are transient. The disk is thus taken to consist primarily of "diffuse" gas, in the sense that only a small fraction of the mass at any time lies in bound clumps having gravitational potential large compared to the mean midplane value in the disk. With an effectively planeparallel distribution of mean gas density, the mean gravitational potential depends only on the distance from the midplane $z$. The time-averaged, horizontally averaged vertical momentum equation (e.g., Boulares \& Cox 1990; Piontek \& Ostriker 2007; Koyama \& Ostriker 2009b; Ostriker et al. 2010) may then be vertically integrated, yielding the result that the total momentum flux through the disk must be equal to the total vertical weight of the overlying gas.

At the midplane, the weight includes a self-gravitational term,

$$
\int_{0}^{\infty} \rho \frac{d \Phi_{g}}{d z} d z=\frac{1}{8 \pi G} \int_{0}^{\infty} \frac{d\left(\frac{d \Phi_{g}}{d z}\right)^{2}}{d z} d z=\frac{\pi G \Sigma^{2}}{2}
$$

where we have used $\rho=(4 \pi G)^{-1} d^{2} \Phi_{g} / d z^{2}$ and $\left|d \Phi_{g} / d z\right|_{\infty}=$ $2 \pi G \Sigma$ for a slab; and a term arising from the external bulge potential, ${ }^{3}$

$$
\int_{0}^{\infty} \rho \frac{d \Phi_{b}}{d z} d z=\frac{4 \pi G \rho_{b}}{3} \int_{0}^{\infty} \rho z d z \equiv \frac{2 \pi \zeta_{d} G \rho_{b} \Sigma^{2}}{3 \rho_{0}} .
$$

Here, $\rho(z)$ is the mean (area-weighted) density of the gas at height $z$ and $\rho_{0}$ is the midplane value. The value of the numerical coefficient $\zeta_{d}$ is insensitive to the exact vertical distribution of the gas, equaling $1 / \pi$ for a Gaussian (when $\Phi_{b}$ dominates) and $\ln (2) / 2$ for a sech ${ }^{2}$ distribution (when $\Phi_{g}$ dominates); $\zeta_{d} \approx 0.33$ within $5 \%$ for these distributions. Note that the self-gravitational weight is independent of the midplane gas density, whereas the weight in the external bulge potential is proportional to $\rho_{b} / \rho_{0}$.

The difference in momentum flux between the disk midplane and the height $z_{\max }$ at which $\rho \rightarrow 0$ includes thermal and turbulent terms, $P_{\text {th }}=\rho_{0}\left\langle v_{\text {th }}^{2}\right\rangle$ and $P_{\text {turb }}=\rho_{0}\left\langle v_{z}^{2}\right\rangle$, where $\left\langle v_{z}^{2}\right\rangle$ is equal to $1 / 3$ of the total turbulent velocity variance $v_{\text {turb }}^{2}$ if it is isotropic. The thermal and turbulent terms can be combined as a single midplane kinetic pressure $\rho_{0} \sigma_{z}^{2}$, where $\sigma_{z}$ is the observed velocity dispersion for a face-on disk. Note that $P_{\text {th }}$ corresponds to a volume-weighted mean pressure and $\left\langle v_{\mathrm{th}}^{2}\right\rangle$ corresponds to a mass-weighted average of thermal speeds of different components: if $M_{\text {total }}=\sum_{i} M_{i}$ and $V_{\text {total }}=\sum_{i} V_{i}$ for mass and volume with $\rho_{i}=M_{i} / V_{i}=$ $P_{\text {th }, \mathrm{i}} / v_{\text {th,i }}^{2}$, then $P_{\text {th }} \equiv \sum_{i} V_{i} P_{\text {th }, \mathrm{i}} / V_{\text {total }}=\sum_{i} M_{i} v_{\text {th, } \mathrm{i}}^{2} / V_{\text {total }}=$ $\rho_{0} \sum_{i}\left(M_{i} / M_{\text {total }}\right) v_{\text {th,i }}^{2} \equiv \rho_{0}\left\langle v_{\text {th }}^{2}\right\rangle$. For molecule-dominated regions, the rms thermal speed $\left\langle v_{\mathrm{th}}\right\rangle^{1 / 2} \lesssim 1 \mathrm{~km} \mathrm{~s}^{-1}$ if $T \lesssim 100 \mathrm{~K}$,

\footnotetext{
3 Note that the expression (2) for a stellar bulge differs from the corresponding equation for a stellar disk in Ostriker et al. (2010) by a factor of $1 / 3$.
}

whereas observed velocity dispersions are at least several $\mathrm{km} \mathrm{s}^{-1}$ so that $\left\langle v_{\mathrm{th}}^{2}\right\rangle \ll\left\langle v_{z}^{2}\right\rangle \approx \sigma_{z}^{2}$. For convenience, we shall use $v_{z}$ to denote the rms value $\left\langle v_{z}^{2}\right\rangle^{1 / 2}$.

The momentum flux difference also contains magnetic terms, $\Delta P_{\text {mag }} \equiv \Delta\left(B_{x}^{2}+B_{y}^{2}-B_{z}^{2}\right) / 8 \pi$, where $\Delta$ denotes the difference between midplane values and values at the surface of the neutral-gas disk. Numerical simulations of highly supersonic magnetohydrodynamic turbulence (e.g., Stone et al. 1998; Mac Low et al. 1998) have shown that the magnetic energy is typically smaller than the turbulent energy by a factor of $\sim 2-3$, so that $B_{\text {midplane }}^{2} /(8 \pi) \lesssim \rho_{0}\left\langle v_{z}^{2}\right\rangle$; this is also consistent with observations (Ferrière 2001). Since the magnetic scale height $L_{B}$ is typically large compared to the semi-thickness $H \equiv \Sigma / \rho_{0}$ of the neutral-gas layer in galactic disks (Tüllmann et al. 2000; Heesen et al. 2009), the ratio $\Delta P_{\mathrm{mag}} / P_{\mathrm{mag}} \sim H / L_{B} \ll 1$, so that the resulting magnetic contribution to the momentum flux difference will be small $\left(\lesssim\left[H / L_{B}\right] \rho_{0}\left\langle v_{z}^{2}\right\rangle\right)$. Cosmic rays and radiation may also contribute to the momentum flux difference, with respective terms $\Delta P_{\mathrm{cr}}$ and $\Delta P_{\text {rad }}$. Including all terms, the total momentum flux corresponds to an effective midplane pressure $P_{\text {eff }} \equiv P_{\text {turb }}+P_{\text {th }}+\Delta P_{\text {mag }}+\Delta P_{\text {cr }}+\Delta P_{\text {rad }}$.

By equating $P_{\text {eff }}$ with the total weight (the sum of Equations (1) and (2)), we obtain

$$
\begin{aligned}
P_{\mathrm{eff}} & \equiv P_{\mathrm{turb}}+P_{\mathrm{th}}+\Delta P_{\mathrm{mag}}+\Delta P_{\mathrm{cr}}+\Delta P_{\mathrm{rad}} \equiv \rho_{0} \sigma_{z}^{2}(1+\mathcal{R}) \\
& =\frac{\pi G \Sigma^{2}}{2}(1+\chi)
\end{aligned}
$$

Here,

$$
\mathcal{R} \equiv \frac{\Delta P_{\mathrm{mag}}+\Delta P_{\mathrm{cr}}+\Delta P_{\mathrm{rad}}}{\rho_{0} \sigma_{z}^{2}}
$$

characterizes the contribution to vertical support from nonkinetic compared to kinetic terms. The term $\chi \equiv 4 \zeta_{d} \rho_{b} /\left(3 \rho_{0}\right)$ represents the contribution to the gas disk's weight from the external bulge potential compared to the gas disk's selfgravitational weight. Since the midplane gas density is given by $\rho_{0}=4 \zeta_{d} \rho_{b} /(3 \chi)$, the vertical equilibrium Equation (3) is a quadratic in $\chi$ that can be solved to obtain

$$
\chi=\frac{2 C(1+\mathcal{R})}{1+[1+4 C(1+\mathcal{R})]^{1 / 2}},
$$

where $C \equiv 8 \zeta_{d} \rho_{b} \sigma_{z}^{2} /\left(3 \pi G \Sigma^{2}\right)$ depends only on large-scale properties of the system. Note that $C^{1 / 2}$ is approximately equal to the ratio of scale heights for a disk-only potential compared to a bulge-only potential, so that $C \ll 1$ if the gas disk dominates vertical gravity and $C \gg 1$ if the bulge dominates vertical gravity.

Equation (5) can also be rearranged to yield $C=\chi(1+\chi) /$ $(1+\mathcal{R})$; when $\mathcal{R}, C \ll 1, \chi \approx C$. Using $\rho_{b}=3 \Omega^{2} /(4 \pi G), C=$ $2 \zeta_{d} \mathcal{W}^{2} \approx 0.7 \mathcal{W}^{2}$ in terms of the quantity $\mathcal{W} \equiv\left(\sigma_{z} \Omega\right) /(\pi G \Sigma)$. The parameter $\mathcal{W}$ is the analog of the Toomre (1964) parameter $Q \equiv \kappa \sigma_{R} /(\pi G \Sigma)$ for vertical rather than horizontal oscillation frequency and velocity dispersion; i.e., $\mathcal{W}=Q \sigma_{z} /\left(2 \sigma_{R}\right)$ for epicyclic frequency $\kappa=2 \Omega$. Thus, if $Q \sim 1-2, \mathcal{R} \ll 1$, and $\sigma_{z} / \sigma_{R} \lesssim 1$, then $C \lesssim 0.2-0.7$ and $\chi \lesssim 0.2-0.5$ so that self-gravity dominates the external (stellar bulge) gravity in controlling the vertical pressure, and $\rho_{0} \gtrsim \rho_{b}$. Alternatively,

$$
Q=\frac{\sigma_{R}}{\sigma_{z}}\left(\frac{2}{\zeta_{d}}\right)^{1 / 2}\left[\frac{\chi(1+\chi)}{1+\mathcal{R}}\right]^{1 / 2}
$$


so that for $\mathcal{R} \ll 1$ and $\chi \lesssim 1, Q \lesssim 3 \chi^{1 / 2} \sigma_{R} / \sigma_{z}$. Also, note that the bulge potential generally dominates orbital motion (even if the gas potential dominates vertical motion), with a factor of $\sim(R / H) C$ for the stellar versus gas contributions to $\Omega^{2}$.

If star formation is self-regulated via momentum inputs, we expect the turbulent portion of the vertical momentum flux through the disk, $\rho_{0}\left\langle v_{z}^{2}\right\rangle$, to be comparable to the total vertical momentum per unit time per unit area that is injected in the gas by feedback from star formation. For isotropic momentum $p_{*}$ injected per massive star near the midplane, averaging over spherical shells yields a vertical component injected on each side of the disk of $p_{*} / 4$. We shall therefore adopt the relation

$$
P_{\text {turb }}=\rho_{0}\left\langle v_{z}^{2}\right\rangle=f_{p} \frac{p_{*}}{4 m_{*}} \Sigma_{\mathrm{SFR}}
$$

where $m_{*}$ is the total mass in stars formed per massive star. The ratio $p_{*} / m_{*}$ is simply the mean radial momentum injected in the ISM per unit mass of stars formed. We have introduced a factor $f_{p}$ to parameterize the dependence on the details of turbulent injection and dissipation. The value of $f_{p}$ is expected to vary between 1 (for strong dissipation) and 2 (for weak dissipation; this is the limit for a stream of particles that is injected vertically and conserves energy as the particles fall back to the midplane).

The kinetic momentum injected in the ISM per massive star can have contributions from a number of sources, including expanding H II regions, stellar winds, and supernovae (e.g., Norman \& Ferrara 1996; Mac Low \& Klessen 2004). If star clusters are born in sufficiently optically thick regions with deep gravitational potential wells, radiation pressure also becomes important in accelerating the residual gas to high velocities (Murray et al. 2010; Krumholz \& Matzner 2009). Matzner (2002) estimates the momentum injected by an expanding $\mathrm{H}$ II region for a given source of ionizing luminosity and ambient density within a GMC, finding a ratio of momentum-to-mass $p_{*} / m_{*} \sim 200-300 \mathrm{~km} \mathrm{~s}^{-1}$ for clouds of mass $\sim 10^{5}-10^{7} M_{\odot}$. Using the results of Matzner (2002), it can be shown that $p_{*} / m_{*} \propto M_{\mathrm{GMC}}^{-4 / 7} R_{\mathrm{GMC}}^{3 / 7} \varepsilon_{\mathrm{GMC}}^{-3 / 7}$ for $M_{\mathrm{GMC}}, R_{\mathrm{GMC}}$, and $\varepsilon_{\mathrm{GMC}}$ the mass, radius, and integrated star-forming efficiency of a GMC, so that GMCs with escape speeds $\left(G M_{\mathrm{GMC}} / R_{\mathrm{GMC}}\right)^{1 / 2}>$ $9 \mathrm{~km} \mathrm{~s}^{-1}$ or $\varepsilon_{\mathrm{GMC}}>0.01$ would have $p_{*} / m_{*} \lesssim 200 \mathrm{~km} \mathrm{~s}^{-1}$. Radiation pressure is most important when gas is concentrated near a central stellar cluster and the efficiency is high (see Appendix A), so that the value of $p_{*} / m_{*}$ will be comparable to the escape speed from the (super)cluster that forms-up to $\sim 100 \mathrm{~km} \mathrm{~s}^{-1}$ for the most extreme clusters (McCrady et al. 2003; Maraston et al. 2004; Overzier et al. 2009).

Although radiation and ionized-gas momentum inputs are important for destroying individual gravitationally bound GMCs (thereby limiting star formation), supernovae are likely the most important feedback mechanism for driving turbulence in the ISM as a whole (Spitzer 1978). For supernova energy of $10^{51} \mathrm{erg}$ and ambient density $10^{3}-1 \mathrm{~cm}^{-3}$, the momentum injection per event is $p_{*} \approx(2-5) \times 10^{5} M_{\odot} \mathrm{km} \mathrm{s}^{-1}$ (Chevalier 1974; Cioffi et al. 1988). If multiple supernovae combine to drive a single expanding shell, stellar winds contribute as well, but this increases the total energy (and momentum, which is approximately linear in the input energy) per unit mass by less than $20 \%$ (Leitherer et al. 1999). For a Kroupa (2001) initial mass function (IMF), the total mass in stars per high mass star $\left(M>8 M_{\odot}\right)$ is $m_{*}=100 M_{\odot}$, so we shall adopt $p_{*} / m_{*}=$ $3000 \mathrm{~km} \mathrm{~s}^{-1}$ from supernovae as our fiducial numerical value.

Under the assumption that cosmic rays are accelerated by supernova blast waves and diffuse vertically out of the disk, the momentum flux contribution from cosmic rays will be proportional to that from supernovae, with

$$
\Delta P_{\mathrm{cr}}=\frac{H}{L_{\mathrm{cr}}} \frac{E_{\mathrm{SN}} \zeta_{\mathrm{cr}}}{2 v_{A} m_{*}} \Sigma_{\mathrm{SFR}}
$$

Here, $\zeta_{\mathrm{cr}}$ is the efficiency of cosmic-ray production per supernova of energy $E_{\mathrm{SN}}$, and we have assumed that the cosmic-ray fluid streams at speed $v_{A}$. The mean free path of cosmic rays, $L_{\text {cr }}$, is likely comparable to the magnetic scale height $L_{B}$ (Yan \& Lazarian 2008). Taking $\zeta_{\mathrm{cr}} \sim 0.1$ (Reynolds 2008), $v_{A} \sim$ $10 \mathrm{~km} \mathrm{~s}^{-1}$, and $E_{\mathrm{SN}}=10^{51} \mathrm{erg},\left(E_{\mathrm{SN}} \zeta_{\mathrm{cr}}\right) /\left(2 v_{A} m_{*}\right)=$ $2500 \mathrm{~km} \mathrm{~s}^{-1}$; this is comparable to the kinetic input $p_{*} / m_{*}$ from supernovae. Since the neutral-disk thickness is small compared to the magnetic scale height, however, $H / L_{\mathrm{cr}} \sim H / L_{B} \ll 1$ and the vertical support of the neutral disk from cosmic rays is small compared to that from turbulence given in Equation (7), $\Delta P_{\mathrm{cr}} /\left(\rho_{0}\left\langle v_{z}^{2}\right\rangle\right) \sim H / L_{\mathrm{cr}} \ll 1$, similar to the situation for magnetic support $\Delta P_{\text {mag }} /\left(\rho_{0}\left\langle v_{z}^{2}\right\rangle\right) \sim H / L_{\mathrm{B}} \ll 1$.

Radiation pressure is important to vertical support of the disk if it is optically thick to reprocessed infrared (IR) radiation (Thompson et al. 2005). Assuming uniformly distributed sources at the disk midplane, the radiation pressure term is

$$
\Delta P_{\mathrm{rad}}=\frac{\kappa_{\mathrm{IR}} \Sigma}{2} \frac{F_{\mathrm{rad}}}{2 c}=\frac{\epsilon_{*} c \kappa_{\mathrm{IR}} \Sigma}{4} \Sigma_{\mathrm{SFR}}
$$

Here, $\kappa_{\mathrm{IR}}$ is the mean opacity, $F_{\text {rad }}$ is the total luminosity per unit area produced by the disk (half is radiated from each side), $c$ is the speed of light, and based on a standard Starburst99 model (Leitherer et al. 1999), $\epsilon_{*} \equiv L_{*} /\left(c^{2} \dot{M}_{*}\right) \approx 6.2 \times 10^{-4}$ is the mass-to-radiation energy conversion efficiency by stars for a Kroupa (2001) IMF in steady state (applicable if the starburst duration is $\gtrsim 10^{7} \mathrm{yr}$ ). The radiation pressure term becomes comparable to the kinetic term driven by supernovae (Equation (7)) if $\epsilon_{*} c \kappa_{\mathrm{IR}} \Sigma$ approaches $p_{*} / m_{*}$.

Retaining just the turbulence and radiation pressure terms in Equation (3), and using Equations (7) and (9), the surface density of star formation is given by

$$
\Sigma_{\mathrm{SFR}}=\frac{2 \pi}{f_{P}} \frac{(1+\chi)}{1+\tau / \tau_{*}} \frac{m_{*} G \Sigma^{2}}{p_{*}}
$$

Here,

$$
\tau_{*} \equiv \frac{f_{p} p_{*}}{\epsilon_{*} m_{*} c}=16 f_{p}\left(\frac{p_{*} / m_{*}}{3000 \mathrm{~km} \mathrm{~s}^{-1}}\right)\left(\frac{\epsilon_{*}}{6.2 \times 10^{-4}}\right)^{-1}
$$

and the optical depth through the disk is

$$
\tau \equiv \kappa_{\mathrm{IR}} \Sigma=0.21\left(\frac{\kappa_{\mathrm{IR}}}{10 \mathrm{~cm}^{2} \mathrm{~g}^{-1}}\right)\left(\frac{\Sigma}{100 M_{\odot} \mathrm{pc}^{-2}}\right) .
$$

Trapped radiation begins to affect the star formation rate when $\tau / \tau_{*} \gtrsim 1$, for very high gaseous surface densities. ${ }^{4}$ Note that in Equation (4), $\mathcal{R} \rightarrow \tau / \tau_{*}$ if radiation dominates over the

\footnotetext{
4 Streaming radiation, as well as diffusing radiation, injects momentum to the disk when it is absorbed. The maximum momentum flux that can be injected to each side of the disk by streaming stellar radiation (including ionizing and far-UV radiation before it is reprocessed to IR) is the input value, $F_{\text {rad }} /(2 c)$. The maximum effect of streaming radiation on self-regulating disk star formation is obtained by replacing $\kappa_{\mathrm{IR}} \Sigma / 2=\tau / 2$ by 1 in Equation (9), so that $\tau / \tau_{*} \rightarrow 2 / \tau_{*}=2 \epsilon_{*} m_{*} c /\left(f_{p} p_{*}\right)$ in Equation (10). Using the fiducial value of $\tau_{*}$ given in Equation (11), the maximum effect of momentum input from streaming stellar radiation is $\sim 10 \%$ compared to that from supernovae.
} 
magnetic and cosmic-ray terms and turbulence dominates the thermal term.

In the turbulence-dominated regime $\left(\tau / \tau_{*} \ll 1\right)$ for selfregulated disks, the surface density of star formation is

$$
\begin{aligned}
\Sigma_{\mathrm{SFR}, \text { turb }}= & \frac{2 \pi(1+\chi)}{f_{P}} \frac{m_{*} G \Sigma^{2}}{p_{*}} \\
= & 0.092 M_{\odot} \mathrm{kpc}^{-2} \mathrm{yr}^{-1} \frac{(1+\chi)}{f_{p}}\left(\frac{p_{*} / m_{*}}{3000 \mathrm{~km} \mathrm{~s}^{-1}}\right)^{-1} \\
& \times\left(\frac{\Sigma}{100 M_{\odot} \mathrm{pc}^{-2}}\right)^{2} .
\end{aligned}
$$

As noted above, disks that are marginally gravitationally unstable $(Q \lesssim 2$ ) have $\chi \lesssim 0.5$. Thus, turbulence-controlled, self-regulated star formation in galactic centers is expected to follow a scaling relation $\Sigma_{\mathrm{SFR}} \propto \Sigma^{2}$.

At very high surface densities such that $\tau / \tau_{*} \gg 1$ (and assuming $\chi \ll 1$ ), the star formation rate in the radiationpressure-dominated regime is

$$
\begin{aligned}
\Sigma_{\mathrm{SFR}, \mathrm{rad}}= & \frac{2 \pi G \Sigma}{\epsilon_{*} c \kappa_{\mathrm{IR}}} \\
= & 710 M_{\odot} \mathrm{kpc}^{-2} \mathrm{yr}^{-1}\left(\frac{\epsilon_{*}}{6.2 \times 10^{-4}}\right)^{-1} \\
& \times\left(\frac{\kappa_{\mathrm{IR}}}{10 \mathrm{~cm}^{2} \mathrm{~g}^{-1}}\right)^{-1}\left(\frac{\Sigma}{10^{4} M_{\odot} \mathrm{pc}^{-2}}\right),
\end{aligned}
$$

which is linear rather than quadratic in the gas surface density.

Equation (13) would apply to galactic center regions experiencing all but the most extreme starburst activity, with a transition to the radiation-dominated regime of Equation (14) at $\tau \sim \tau_{*}$, corresponding to a gas surface density

$$
\begin{aligned}
\Sigma_{\text {trans }, \mathrm{rad}}= & 8 \times 10^{3} M_{\odot} \mathrm{pc}^{-2}\left(\frac{\kappa_{\mathrm{IR}}}{10 \mathrm{~cm}^{2} \mathrm{~g}^{-1}}\right)^{-1} \\
& \times\left(\frac{p_{*} / m_{*}}{3000 \mathrm{~km} \mathrm{~s}^{-1}}\right)\left(\frac{\epsilon_{*}}{6.2 \times 10^{-4}}\right)^{-1} .
\end{aligned}
$$

The self-regulated turbulent galactic-center regime of Equation (13) connects with the "mid-disk" regime, where internal GMC processes control star formation (see Section 1), at lower surface densities. Setting Equation (13) equal to $\Sigma_{\mathrm{SFR}}=\Sigma / t_{\mathrm{SF}, \mathrm{GMC}}$ with $t_{\mathrm{SF}, \mathrm{GMC}}$ comparable to the empirical mid-disk value $t_{\mathrm{SF}, \mathrm{mol}} \sim 2$ Gyr (Bigiel et al. 2008; Blanc et al. 2009) and assuming $\chi \ll 1$, the nominal transition to the middisk regime is at

$$
\Sigma_{\text {trans }, \mathrm{GMC}}=54 M_{\odot} \mathrm{pc}^{-2} f_{p}\left(\frac{p_{*} / m_{*}}{3000 \mathrm{~km} \mathrm{~s}^{-1}}\right)\left(\frac{t_{\mathrm{SF}, \mathrm{GMC}}}{2 \mathrm{Gyr}}\right)^{-1}
$$

Physically, this transition occurs because at low surface densities, the energy input from star formation is insufficient to prevent "diffuse" molecular gas from becoming concentrated in individual gravitationally bound GMCs.

\section{COMPARISON OF $\Sigma_{\text {SFR }}$ VERSUS $\Sigma$ TO NUMERICAL SIMULATIONS}

To initiate a numerical study of star formation under high surface density conditions, we begin with a very simple computational model, which nevertheless includes sufficient ingredients that we are able to investigate self-regulation of star formation in turbulence-dominated disks. Our simulation domain represents a local region within a starburst disk, which we resolve both vertically and horizontally. The domain is rotating with the disk, so we include centrifugal and Coriolis forces; we assume a solid-body rotation curve, so there is no large-scale rotational shear within the domain. We assume that the gas cools efficiently to maintain an approximately constant (low) temperature, so we adopt an isothermal equation of state with sound speed $c_{s}=(P / \rho)^{1 / 2}$. For this first study, we neglect magnetic fields. Thus, the dynamical equations we solve are

$$
\begin{gathered}
\frac{\partial \rho}{\partial t}+\nabla \cdot(\rho \mathbf{v})=0 \\
\frac{\partial \mathbf{v}}{\partial t}+\mathbf{v} \cdot \nabla \mathbf{v}=-\frac{1}{\rho} \nabla P-2 \boldsymbol{\Omega} \times \mathbf{v}-\nabla \Phi_{g}+\mathbf{g}_{\text {ext }},
\end{gathered}
$$

and

$$
\nabla^{2} \Phi_{g}=4 \pi G \rho
$$

(e.g., Kim et al. 2002, taking dimensionless shear parameter $q=0$ ). For a spherical bulge, the vertical gravitational field is given by $\mathbf{g}_{\text {ext }}=-\Omega^{2} z \hat{z}$.

We integrate the dynamical equations using a version of the Athena code (Stone et al. 2008), which employs a single-step, directionally unsplit Godunov scheme (Gardiner \& Stone 2005, 2008) to obtain conservative, second-order accurate solutions. The Poisson equation is solved using the Fourier transform method of Koyama \& Ostriker (2009a), suitable for disk problems that are treated as having open boundary conditions in the vertical $(\hat{z})$ direction and periodic boundary conditions in the horizontal directions. The equations above can be solved either for a three-dimensional domain, or a two-dimensional domain representing a radial-vertical slice through the disk (e.g., Kim et al. 2006; Koyama \& Ostriker 2009a). Two-dimensional radialvertical models, which we adopt here, capture the physics of the competition between turbulent driving and gravitational settling in the vertical direction, and allow much more extensive initial exploration of parameters than is possible for three-dimensional models (which we intend to pursue next).

Motivated by observations showing a linear relation between very dense gas and star formation (e.g., Gao \& Solomon 2004; $\mathrm{Wu}$ et al. 2005, 2010), we assume that star formation takes place in gas above a density threshold $\rho_{\text {th }}=\mu n_{\text {th }}$ at a fixed efficiency per free-fall time $\varepsilon_{\mathrm{ff}}\left(n_{\mathrm{th}}\right)$; we also assume that the total mass in stars formed per massive star is $m_{*}$ and that each massive star injects a momentum $p_{*}$ into the ISM. Thus, when $n \geqslant n_{\text {th }}$ in a given zone containing total mass $m$, the probability of a massive star formation event occurring within a given time step $\Delta t$ is set to be $P=\Delta t \varepsilon_{\mathrm{ff}}\left(n_{\mathrm{th}}\right) m /\left[m_{*} t_{\mathrm{ff}}(\rho)\right]$. Here, $t_{\mathrm{ff}}(\rho)=[3 \pi /(32 G \rho)]^{1 / 2}$ is the gravitational free-fall time at density $\rho$. For every zone where a massive star formation event occurs, we inject a total momentum $p_{*}$ into the gas in a spherical region of radius $R_{\text {sh }}$ surrounding the event. For every feedback event associated with a massive star, the tally of the total mass in stars formed is increased by $m_{*}$, although we do not remove any gas from the grid (in order to maintain constant $\Sigma)$. The computed surface star formation rate $\Sigma_{\mathrm{SFR}}$ is the mass in stars formed per unit time within the domain, divided by the horizontal area.

A description of the detailed numerical methods and the results of a full parameter survey (varying $\Sigma, \Omega, c_{s}, \varepsilon_{\mathrm{ff}}\left(n_{\mathrm{th}}\right)$, $p_{*} / m_{*}, R_{\mathrm{sh}}, n_{\mathrm{th}}$, domain size $L_{x}, L_{z}$, and numerical resolution), will be presented separately (R. Shetty \& E. C. Ostriker 2011, in preparation). Here, since we are interested in comparing 

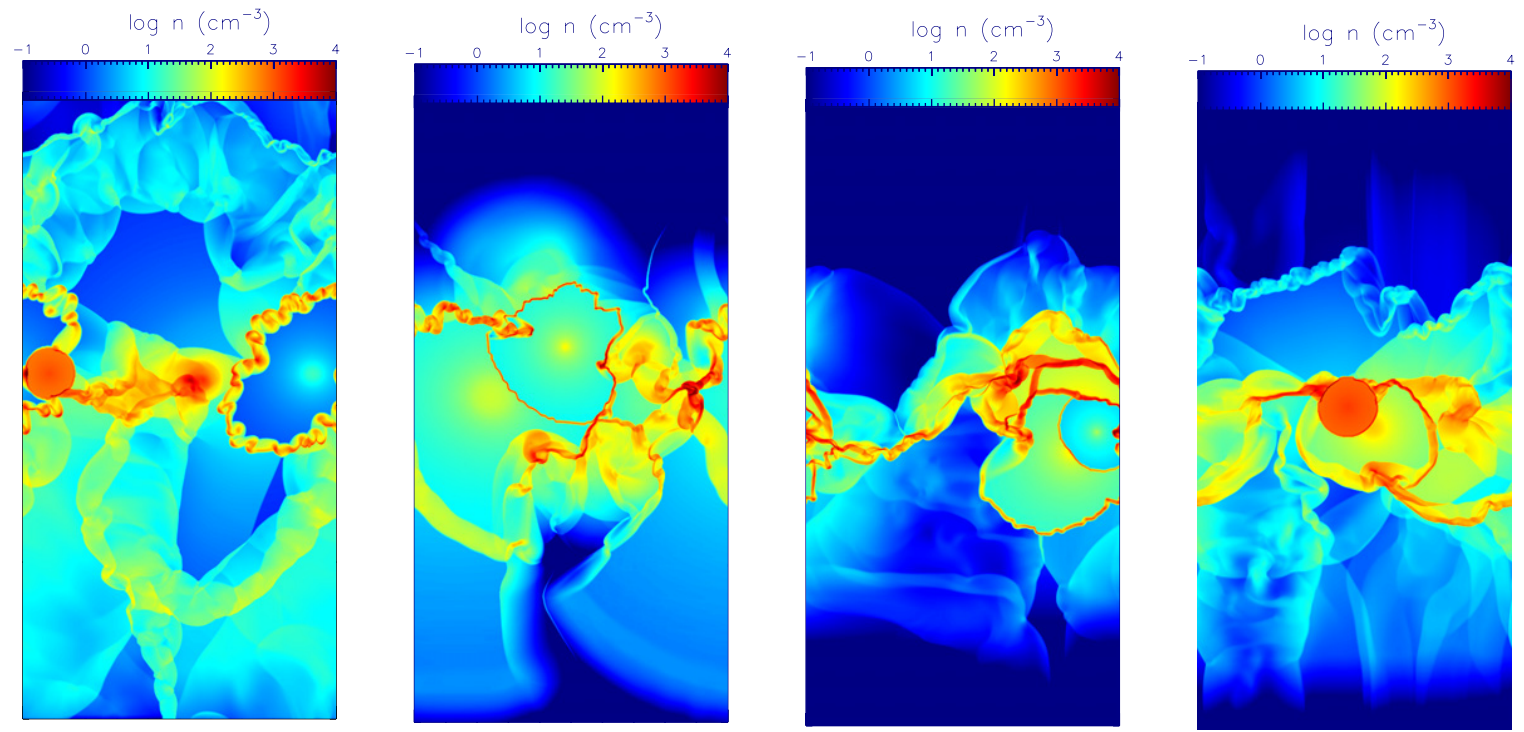

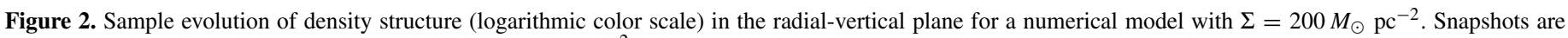
separated by 5 Myr. The domain size is $L_{x} \times L_{z}=60 \times 120 \mathrm{pc}^{2}$.

(A color version of this figure is available in the online journal.)

to Equation (13) to test the idea of turbulent self-regulation, we focus on the dependence of the star formation rate $\Sigma_{\mathrm{SFR}}$ on the gas surface density $\Sigma$. We fix $m_{*}=100 M_{\odot}, p_{*}=$ $3 \times 10^{5} M_{\odot} \mathrm{km} \mathrm{s}^{-1}, R_{\mathrm{sh}}=5 \mathrm{pc}, c_{s}=2 \mathrm{~km} \mathrm{~s}^{-1}$, and set the angular velocity to $\Omega=\operatorname{Myr}^{-1}\left(\Sigma / 10^{3} M_{\odot} \mathrm{pc}^{-2}\right.$ ) (so that the Toomre parameter would be unity for a velocity dispersion of $7 \mathrm{~km} \mathrm{~s}^{-1}$ ). Note that the value of $c_{s}$ we use is larger than would be provided by thermal pressure at temperatures $\sim 10-100 \mathrm{~K}$; without magnetic fields (which are neglected in this study), shocks would become unphysically strong if $c_{s}$ is much lower. Turbulent energy is still far larger than thermal energy for the parameter range under consideration, so that the disk thickness is controlled by feedback-driven turbulence (see Section 5 and R. Shetty \& E. C. Ostriker 2011, in preparation). The models are integrated for $200 \mathrm{Myr}$; a quasi-steady state typically requires only a few tens of Myr to be established, for the parameter regime $\Sigma \gtrsim 100 M_{\odot} \mathrm{pc}^{-2}$ under investigation.

Figure 2 shows an example of typical density structure snapshots in the radial-vertical plane, from a model with $\Sigma=$ $200 M_{\odot} \mathrm{pc}^{-2}$, taking $n_{\mathrm{th}}=5 \times 10^{3} \mathrm{~cm}^{-3}$ and $\varepsilon_{\mathrm{ff}}\left(n_{\mathrm{th}}\right)=0.01$. This density threshold and efficiency factor are chosen based on observations of Milky Way and extragalactic gas and star formation (Krumholz \& Tan 2007; Evans et al. 2009; Lada et al. 2010; Heiderman et al. 2010; see also Section 5). As Figure 2 shows, the structure is highly inhomogeneous due to the dominance of turbulence, which shocks and compresses the gas. Effects of recent star formation events are seen as dense circular regions; these drive expanding shells, which are also evident. Although structure is irregular and highly dynamic, gas is preferentially concentrated toward the midplane due to gravity; this is where star formation events take place, in the densest gas. All of our models show similar structure and evolution, with repeated local collapse events and feedbackdriven turbulent excitation. In some of our simulations with a sufficiently large radial domain, we find that the gas may collapse into a single condensation that the feedback is unable to redisperse, because the gravitational potential well is too deep. As we discuss in Section 5 and the Appendix, in real galaxies radiation pressure may play a role in dispersing strongly bound clouds and superclouds. Since radiation is not included in the current simulations, we consider only models that do not suffer this kind of global collapse.

In spite of their highly dynamic behavior, our simulations show that a quasi-steady state is established in which the vertical weight of the gas is approximately balanced by turbulent pressure, which itself is driven by momentum injection associated with massive star formation. For the same model shown in Figure 2, Figure 3 shows the evolution of the turbulent momentum flux in the vertical direction $\rho_{0} v_{z}^{2}$ and the characteristic vertical momentum injection rate to each side of the disk from star formation $0.25\left(p_{*} / m_{*}\right) \Sigma_{\mathrm{SFR}}$, as well as the mean vertical weight of the gas disk $0.5 \pi G \Sigma^{2}(1+\chi)$ (the value of $\chi \sim 0.1$ for this model); the respective time-averaged values of these quantities are 357, 209, and $305 M_{\odot} \mathrm{km} \mathrm{s}^{-1} \mathrm{pc}^{-2} \mathrm{Myr}^{-1}$. From Equation (7), $f_{p} \sim 1.7$, consistent with the expectation that the turbulent pressure is comparable to the momentum injection rate per unit area from star formation. Note that the difference between the mean momentum flux and the mean weight is due to a combination of stochastic effects and non-plane-parallel variations in the gravitational potential. Our other models show similar temporal behavior for the various momentum flux terms.

Figure 4 shows the time-averaged values of $\Sigma_{\mathrm{SFR}}$ measured from a set of simulations with $\Sigma=100,200,400,800 M_{\odot} \mathrm{pc}^{-2}$. For all models we set $n_{\mathrm{th}}=5 \times 10^{3} \mathrm{~cm}^{-3}$; we show cases with $\varepsilon_{\mathrm{ff}}\left(n_{\mathrm{th}}\right)=0.005,0.01$, and 0.05 . The numerical results are consistent with the prediction given in Equation (13) for momentum-controlled, self-regulated disk star formation. We note that both turbulence and small-scale self-gravity are crucial in determining the star formation rate in these simulations. For all of the models, our adopted $n_{\text {th }}$ exceeds the midplane value of the density for a purely thermally supported slab (with the adopted value $c_{s}=2 \mathrm{~km} \mathrm{~s}^{-1}$ ), so no star formation would take place without localized gravitational collapse. On the other hand, if we had not imposed a high threshold density and had simply assumed a star formation rate equal to $\varepsilon_{\mathrm{ff}} \Sigma / t_{\mathrm{ff}}\left(n_{\text {midplane }}\right)$ for our adopted $c_{s}$ and $\varepsilon_{\mathrm{ff}}=0.01$ (see Section 5), we would have obtained the same scaling with surface density $\Sigma_{\mathrm{SFR}} \propto \Sigma^{2}$ as found with our simulations (and as predicted by Equation (13)), but a much larger coefficient: $\Sigma_{\mathrm{SFR}}=\varepsilon_{\mathrm{ff}} 4 G \Sigma^{2} /\left(\sqrt{3} c_{s}\right)$. For the value $c_{s}=2 \mathrm{~km} \mathrm{~s}^{-1}$ used in the present simulations, this 


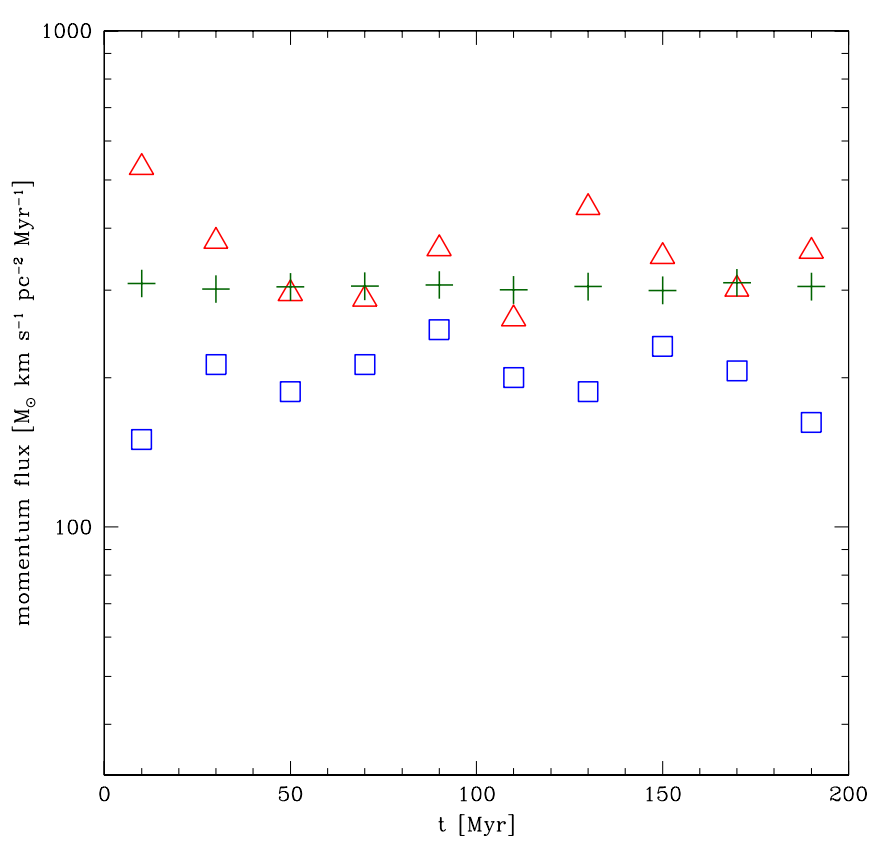

Figure 3. Comparison of characteristic input vertical momentum flux from star formation $0.25\left(p_{*} / m_{*}\right) \Sigma_{\mathrm{SFR}}$ (squares), turbulent pressure in the vertical direction $\rho_{0} v_{z}^{2}$ (triangles), and vertical weight of the gas disk $0.5 \pi G \Sigma^{2}(1+\chi)$ (plusses) for the same model as shown in Figure 2. Each quantity is computed averaging over temporal bins of $20 \mathrm{Myr}$.

(A color version of this figure is available in the online journal.)

would yield a factor of $\sim 6$ larger $\Sigma_{\mathrm{SFR}}$ than Equation (13); lower $c_{s}$ would yield even larger $\Sigma_{\mathrm{SFR}}$. Thus, a simulation that did not include turbulent feedback but allowed gas to cool to low temperature (small $c_{s}$ ) could significantly overestimate the star formation rate. Turbulence driven by star formation keeps the volume-averaged midplane value of the density lower than that of a thermally supported disk, but self-gravitating collapse of shocked, highly overdense local regions still occurs, leading to further (self-regulated) star formation.

\section{COMPARISON OF $\Sigma_{\text {SFR }}$ VERSUS $\Sigma$ TO OBSERVATIONS}

The dependence of $\Sigma_{\text {SFR }}$ on $\Sigma$ predicted by the simple model of Section 2 can be compared to observations. Genzel et al. (2010a) have recently investigated the relationship between molecular gas and star formation in a large sample compiled from both local-universe and high-redshift galaxies. The compilation includes local ULIRGs as well as normal and merging galaxies at $z \sim 1-3$. Gaseous surface densities in this (and other) data sets are based on observations of $\mathrm{CO}$ emission, so it is necessary to convert the integrated line emission, $I_{\mathrm{CO}}$, to a value of $\Sigma$. This conversion is accomplished using a factor $\alpha \equiv \Sigma_{\mathrm{H} 2} / I_{\mathrm{CO}}$ or $X_{\mathrm{CO}} \equiv N_{\mathrm{H} 2} / I_{\mathrm{CO}}$, where $\Sigma_{\mathrm{H} 2}=\Sigma / 1.4$ is the surface density of molecular hydrogen in $M_{\odot} \mathrm{pc}^{-2}$ (the factor 1.4 accounts for helium in the total surface density), $N_{\mathrm{H} 2}$ is the molecular hydrogen column density in $\mathrm{cm}^{-2}$, and $I_{\mathrm{CO}}$ is in units of $\mathrm{K} \mathrm{km} \mathrm{s}^{-1}$ so that $X_{\mathrm{CO}}=6.3 \times 10^{19} \alpha$ in units $\mathrm{cm}^{-2} /\left(\mathrm{K} \mathrm{km} \mathrm{s}^{-1}\right)$ if $\alpha$ has units $M_{\odot} \mathrm{pc}^{-2} /\left(\mathrm{K} \mathrm{km} \mathrm{s}^{-1}\right)$. In Milky Way and Local Group GMCs, values of $\alpha \approx 2-5$ (for the $\mathrm{CO} \mathrm{J}=1-0$ transition) have been inferred based on a variety of empirical techniques (e.g., Blitz et al. 2007), whereas there is evidence that $\alpha$ is significantly lower in ULIRGs and other extreme star-forming systems (e.g., Solomon \& Vanden Bout 2005).

For surface densities $\Sigma \lesssim 100 M_{\odot} \mathrm{pc}^{-2}$ in galaxies, CO emission can be understood as arising primarily from collections of gravitationally bound GMCs with limited variation in individ-

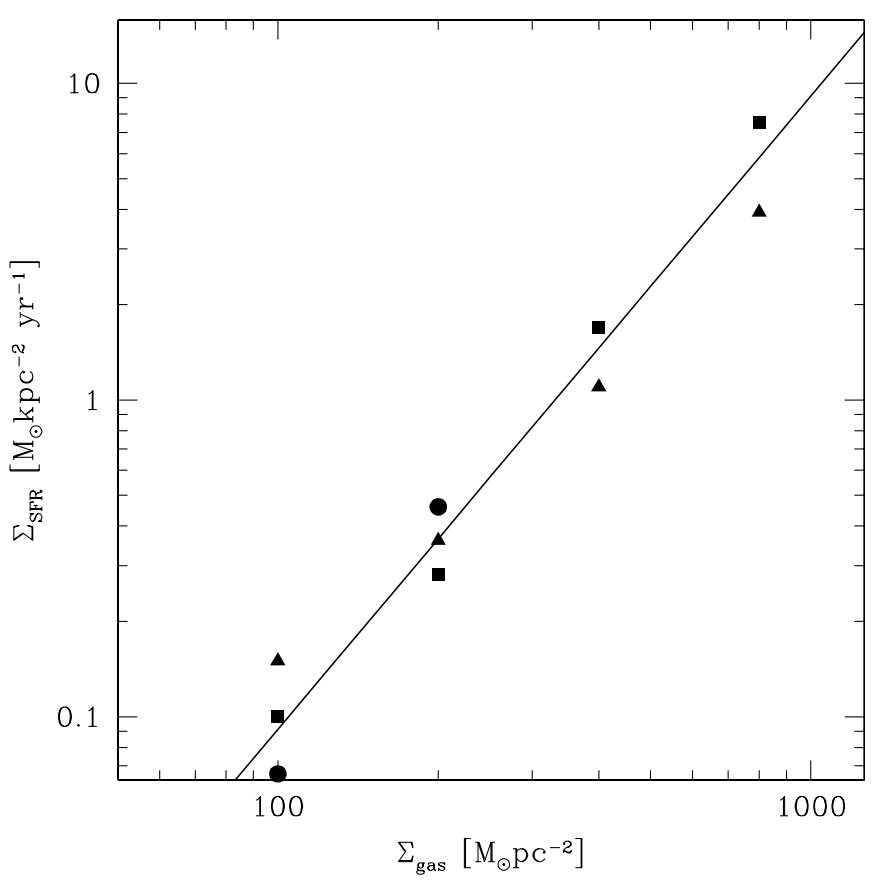

Figure 4. Results of mean measured star formation rate $\Sigma_{\mathrm{SFR}}$ from a set of simulations with a range of gas surface density $\Sigma$ in comparison with the prediction given in Equation (13) (solid line). For the numerical results, triangles have $\epsilon_{\mathrm{ff}}\left(n_{\mathrm{th}}\right)=0.005$, squares have $\epsilon_{\mathrm{ff}}\left(n_{\mathrm{th}}\right)=0.01$, and circles have $\epsilon_{\mathrm{ff}}\left(n_{\mathrm{th}}\right)=0.05$.

ual properties such as total column density and kinetic temperature (Solomon et al. 1987; Bolatto et al. 2008; Heyer et al. 2009), such that there is an approximately constant conversion factor $\alpha$. The standard value of $X_{\mathrm{CO}}=2 \times 10^{20}$ based on $\mathrm{CO}$ emission in the Milky Way (Strong \& Mattox 1996; Dame et al. 2001) corresponds to $\alpha=3$.2. Shetty et al. (2011) have applied radiative transfer to numerical simulations of turbulent clouds, confirming that mean ratio of $N_{\mathrm{H} 2} / I_{\mathrm{CO}}$ is comparable to standard empirically estimated values $X_{\mathrm{CO}}=2 \times 10^{20}$, provided that $A_{V}$ is neither very low nor very high.

For galactic center regions with high surface densities, however, the molecular gas may respond to the overall gravitational potential in the disk rather than being bound in individual GMCs (raising the velocity dispersion), and may be systematically warmer for higher $\Sigma_{\mathrm{SFR}}$. These effects would increase the integrated $\mathrm{CO}$ emission $I_{\mathrm{CO}}$ for a given $\Sigma$, implying a smaller $X_{\mathrm{CO}}$ or $\alpha$ is needed (Solomon et al. 1997). Since the dependence of $\Sigma_{\mathrm{SFR}}$ on $\Sigma$ is nonlinear, gas surface densities may also be overestimated in some cases if the gas is not spatially resolved and the characteristic radius of the IR-emitting starburst is adopted as a surrogate (this spatial mismatch is known to be an issue for high-redshift galaxies; see, e.g., Bothwell et al. 2010). In addition, lack of spatial resolution combined with galactic inclination can broaden observed CO line widths (due to rotation and to radial and/or azimuthal streaming within a beam) beyond the true turbulent velocity width in a local patch of the disk; without detailed modeling, it is not clear how this would affect the conversion between $I_{\mathrm{CO}}$ and $N_{\mathrm{H} 2}$. For ULIRGs with typical $\Sigma \sim 10^{3} M_{\odot} \mathrm{pc}^{-2}$, empirically estimated values are $\alpha \lesssim 1$ (Downes \& Solomon 1998).

From a theoretical point of view, the value of $\alpha$ in a selfregulated starburst system should depend primarily on the disk's gas surface density $\Sigma$, since this sets $\Sigma_{\mathrm{SFR}}$ and thus the gas velocity dispersion and the distributions of temperature and density through star formation feedback. Using numerical 


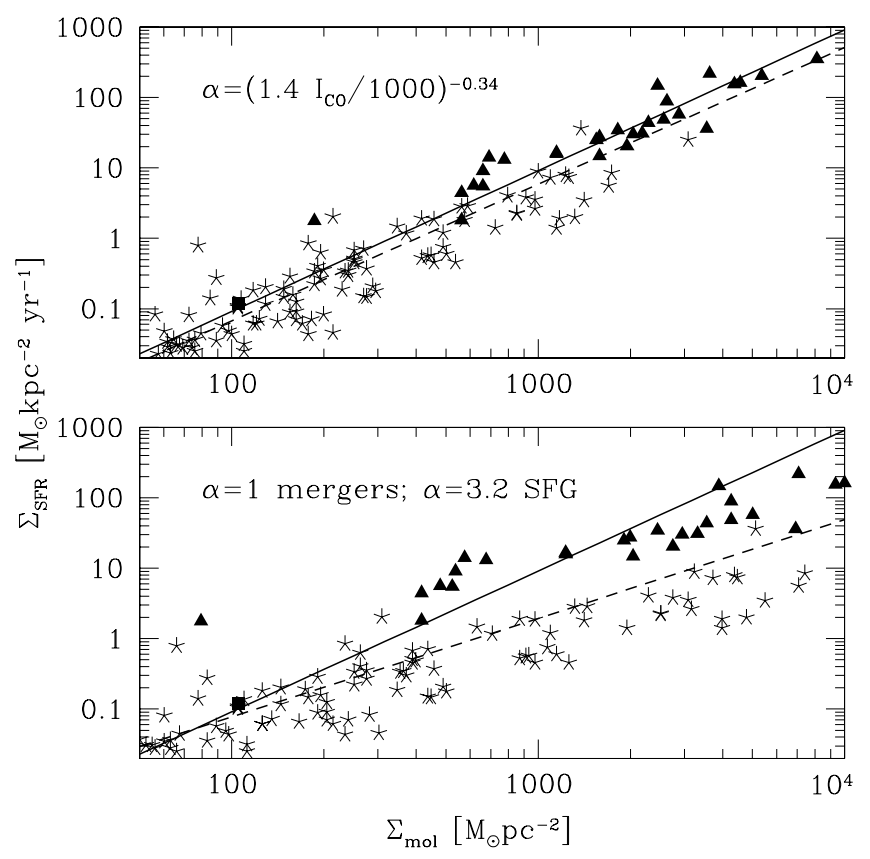

Figure 5. Solution given by Equation (13) for star formation rate $\Sigma_{\text {SFR }}$ vs. gas surface density $\Sigma$ (solid line) in comparison with data compiled in Genzel et al. (2010a). In the lower panel, $\Sigma_{\mathrm{mol}} /\left[M_{\odot} \mathrm{pc}^{-2}\right] \equiv 1.4 \alpha I_{\mathrm{CO}} /\left[\mathrm{K} \mathrm{km} \mathrm{s}^{-1} \mathrm{pc}^{-2}\right]$ is computed assuming $\alpha=3.2$ for star-forming galaxies (stars) and $\alpha=1$ for merger systems (triangles), following Genzel et al. (2010a). In the upper panel, a variable value of $\alpha$ is adopted such that $\alpha=3.2$ for $\Sigma=100 M_{\odot} \mathrm{pc}^{-2}$ and $\alpha=1$ for $\Sigma=1000 M_{\odot} \mathrm{pc}^{-2}$. Dashed lines show the least-squares fit to the data, with slopes 1.4 in the lower panel and 1.9 in the upper panel. The value obtained by Yusef-Zadeh et al. (2009) for the Milky Way center is also shown (squares in both panels).

simulations for a range of disk properties combined with detailed radiative transfer calculations, it will ultimately be possible to obtain predictions for the dependence of $\alpha$ on local conditions. Empirically, a systematic trend for $\alpha$ to decrease with increasing $\Sigma$ is already evident (e.g., Tacconi et al. 2008). The simplest possible relation would be for $I_{\mathrm{CO}}$, and therefore $\alpha$, to depend on $\Sigma$ as a power law. For example, if $\alpha$ is fixed to 1 at $\Sigma=10^{3} M_{\odot} \mathrm{pc}^{2}$ from ULIRG observations, and to 3.2 at $\Sigma=10^{2} M_{\odot} \mathrm{pc}^{2}$ from the limit of "normal" GMCs, a power-law assumption yields $\alpha=(\Sigma / 1000)^{-0.52}=\left(1.4 I_{\mathrm{CO}} / 1000\right)^{-0.34}$.

In Figure 5, we compare the prediction of Equation (13) with the observed relation between $\Sigma$ and $\Sigma_{\text {SFR }}$ from the compilation of Genzel et al. (2010a). Also shown is the surface density and star formation rate in the Milky Way Galactic center, from Yusef-Zadeh et al. (2009). For the theoretical result, we have set $\chi \rightarrow 0$ in Equation (13) from Equation (5), since $C<0.1$ in the observed sample (except for two cases which have $C<0.5$ ) if we assume the vertical velocity dispersion is $\sim 10 \mathrm{~km} \mathrm{~s}^{-1}$. In the presentation of Genzel et al. (2010a), a value $\alpha=1$ is adopted for merger galaxies (LIRGs and ULIRGs), while a value $\alpha=3.2$ is adopted for other systems. In Figure 5, we compare to observations using both these standard conversion factors (lower panel), and using the simple power-law function for $\alpha$ given above (upper panel).

Evidently, the theoretical prediction of Equation (13) follows the data over the full range of surface densities if a $\Sigma$-dependent $\alpha$ is used. If $\alpha=1$ is used for mergers and $\alpha=3.2$ for normal galaxies, Equation (13) agrees with the data at low $\Sigma$, but at high $\Sigma$ there is an offset between the two classes of galaxies, with only the mergers close to the result of Equation (13). Genzel et al. (2010a) and Daddi et al. (2010) previously noted this offset, and suggested that a difference in dynamical timescale $\Omega^{-1}$ may be responsible for the apparent difference in star formation laws. With $\alpha$ depending on $\Sigma$, star formation follows a single relation for both mergers and normal galaxies in the $\Sigma-\Sigma_{\mathrm{SFR}}$ plane, but mergers are preferentially at higher $\Sigma$-which is correlated empirically with higher $\Omega$. Regardless of the value of $\alpha$, star formation is observed to be more efficient in merger systems, based on the ratio $L_{\mathrm{IR}} / L_{\mathrm{CO}}$. Given the characteristically larger values of $I_{\mathrm{CO}}$ and $\Sigma$ for mergers compared to normal galaxies, this is consistent with theoretical expectations that $\Sigma_{\text {SFR }}$ depends nonlinearly on $\Sigma$-in particular, as $\Sigma_{\mathrm{SFR}} \propto \Sigma^{2}$ based on Equation (13).

\section{DISK PROPERTIES IN THE TURBULENCE-DOMINATED REGIME}

In Section 2, we showed that if turbulence driven by star formation feedback dominates other forms of pressure, the equilibrium star formation rate is expected to be proportional to the weight of the ISM. ${ }^{5}$ The force balance (or momentum conservation) argument leads to a relation $\Sigma_{\mathrm{SFR}} \propto \Sigma^{2}$ for gas-dominated regions in a self-regulated steady state, but it says nothing about the internal processes or character of the disk. In order to maintain equilibrium, the disk must internally adjust itself so that stars are produced at the required rate, and this internal arrangement must also be consistent with the detailed dynamics of a self-gravitating, turbulent hydrodynamic (or hydromagnetic) flow.

In a region of mean gas density $\bar{\rho}$ (and where $\rho_{*} \ll \bar{\rho}$ ), the gravitational free-fall time at the mean density, $t_{\mathrm{ff}}(\bar{\rho})=$ $[3 \pi /(32 G \bar{\rho})]^{1 / 2}$, represents a characteristic dynamical time. The star formation rate can then be expressed as $\dot{M}_{*} \equiv$ $\varepsilon_{\mathrm{ff}}(\bar{\rho}) M_{\mathrm{gas}} / t_{\mathrm{ff}}(\bar{\rho})$, where $\varepsilon_{\mathrm{ff}}(\bar{\rho})$ is an efficiency factor that depends on the detailed properties of the gas. Although it is uncertain exactly what controls the rate of localized gas collapse in highly turbulent systems, evidence from current observations (Krumholz \& Tan 2007; Evans et al. 2009), theory (Krumholz \& McKee 2005; Padoan \& Nordlund 2009), and numerical simulations (Padoan \& Nordlund 2009; Rosas-Guevara et al. 2010; Vázquez-Semadeni et al. 2009) suggests that the star formation efficiency factor is low, $\varepsilon_{\mathrm{ff}}(\bar{\rho}) \sim 0.01$, for molecular gas in observed star-forming regions at a range of $\bar{\rho}$. Krumholz \& McKee (2005) proposed that for turbulence-dominated systems, $\varepsilon_{\mathrm{ff}}(\bar{\rho})$ is given by the fraction of mass compressed to densities higher than the mean by a factor $\sim \mathcal{M}^{2}$ for $\mathcal{M} \equiv v_{\text {turb }} / v_{\text {th }}$ the Mach number. Because the density PDF in turbulent regions is a lognormal function of $\mathcal{M}$ (McKee \& Ostriker 2007), Krumholz \& McKee (2005) show that the resulting dependence of the star-forming efficiency on the Mach number is weak, $\varepsilon_{\mathrm{ff}}(\bar{\rho}) \propto \mathcal{M}^{-0.3}$. Thus, if localized collapse to make stars is controlled primarily by turbulence, the overall rate is expected to be governed primarily by the free-fall time on large scales.

In (self-gravitating) disks, it is convenient to use the mean midplane density $\rho_{0}=\mu n_{0}$ as a reference value, so that the star formation rate per unit area can be written as

$$
\Sigma_{\mathrm{SFR}} \equiv \varepsilon_{\mathrm{ff}}\left(\rho_{0}\right) \frac{\Sigma}{t_{\mathrm{ff}}\left(\rho_{0}\right)} .
$$

\footnotetext{
5 The star formation rate is also expected to be proportional to the weight of the ISM if thermal pressure dominates other forms of pressure, in a medium dominated by diffuse atomic gas and heated by young stars (Ostriker et al. 2010). If, however, radiation pressure dominates both turbulent and thermal pressure, the star formation rate would be proportional to the vertical gravitational field rather than the weight (i.e., reduced by a factor $\propto 1 / \tau \propto 1 / \Sigma-c f$. Equations (13) and (14)).
} 
For a Gaussian vertical density distribution, $\bar{\rho}=\rho_{0} / \sqrt{2}$ so that $\varepsilon_{\mathrm{ff}}\left(\rho_{0}\right)=0.8 \varepsilon_{\mathrm{ff}}(\bar{\rho})$. As in Section 2, in adopting the form of Equation (20), we have implicitly assumed that the gas collapses only locally —on scales small compared to the Toomre wavelength (Toomre 1964) $\lambda_{T} \equiv G \Sigma(2 \pi / \kappa)^{2}$ —so that angular momentum and shear do not dominate the dynamics of star formation. In addition, Equation (20) implicitly assumes that the free-fall time at the mean midplane gas density is shorter than the vertical oscillation time in the gravitational field of the stellar bulge; this will be true if gas self-gravity dominates vertical potential gradients.

We note that Equation (20) is not expected to apply in regions of a galactic disk that have a fundamentally multiphase character, in terms of the mass- and volume- fractions of gas at different temperatures. In particular, if most of the ISM's mass is in clouds that are self-gravitating entities isolated from their surroundings, the local density within these self-gravitating, star-forming clouds need not be proportional to the (volumeweighted) mean midplane density of the disk. Using multiphase disk simulations, Koyama \& Ostriker (2009a) indeed found that Equation (20) would yield a different (steeper) star formation law than is obtained by adopting a constant star formation timescale only in very dense (gravitationally bound) gas.

For outer-disk regions dominated in mass by diffuse atomic gas, Ostriker et al. (2010) argued that a key factor regulating star formation is the heating and cooling of the warm, volumefilling medium. Gas that cannot be maintained in thermal equilibrium in the diffuse phase cools and drops out to make gravitationally bound, star-forming clouds. This increases the heating rate (from stellar UV), and decreases the cooling rate (since the density is lower), for the remaining diffuse gas. Mass dropout continues until heating balances cooling. In a state of simultaneous thermal and dynamical equilibrium, the star formation rate for an outer-disk region will still vary inversely with the dynamical time of the dominant gas component, analogous to Equation (20), but the relevant dynamical time is not $t_{\mathrm{ff}}\left(\rho_{0}\right)$. In outer-disk regions, the ISM scale height is generally set by the gravity of stars plus dark matter, rather than the self-gravity of the gas. As a consequence, the gas disk's internal dynamical time $t_{\mathrm{dyn}} \sim H / \sigma_{z}$ (or the timescale for vertical oscillations $\left.\sim 2 \pi H / \sigma_{z}\right)$ varies as $t_{\mathrm{dyn}} \propto\left(G \rho_{*}\right)^{-1 / 2}$ rather than $t_{\mathrm{dyn}} \propto t_{\mathrm{ff}}\left(\rho_{0}\right) \propto\left(G \rho_{0}\right)^{-1 / 2}$, yielding an outer-disk star formation rate $\Sigma_{\mathrm{SFR}} \propto \Sigma \sqrt{\rho_{*}}$ (see Equations (22) and (A16) in Ostriker et al. 2010).

For turbulent, molecular-gas-dominated starburst regions where Equation (20) is hypothesized to apply, the value of $\varepsilon_{\mathrm{ff}}\left(\rho_{0}\right)$ is uncertain, but analysis of observations including nearby star-forming clouds, and both moderate-density and high-density extragalactic molecular gas, is consistent with a range $\varepsilon_{\mathrm{ff}}\left(\rho_{0}\right) \sim 0.003-0.06$ (Krumholz \& Tan 2007; Bigiel et al. 2008; Evans et al. 2009; Blanc et al. 2009). Simulations of turbulent, star-forming disks can also be used to evaluate $\varepsilon_{\mathrm{ff}}\left(\rho_{0}\right)$. The mean midplane density $\rho_{0}$ can be measured and used to obtain a mean value of $t_{\mathrm{ff}}\left(\rho_{0}\right)$; if the value of $\Sigma_{\mathrm{SFR}}$ measured in the simulations is multiplied by the measured $t_{\mathrm{ff}}\left(\rho_{0}\right)$ and divided by $\Sigma$, the result is a numerical evaluation of $\varepsilon_{\mathrm{ff}}\left(\rho_{0}\right)$. For the numerical simulations described in Section 3, the models yield a range $\varepsilon_{\mathrm{ff}}\left(\rho_{0}\right) \sim 0.004-0.01$ (for further discussion, see R. Shetty \& E. C. Ostriker 2010, in preparation). As a fiducial value, we shall adopt $\varepsilon_{\mathrm{ff}}\left(\rho_{0}\right)=0.005$, but it is important to keep in mind that further observations and simulations are needed to determine this value (and any dependence on parameters) more conclusively.
A disk that is in vertical equilibrium will obey Equation (3); assuming gas thermal+turbulent pressure exceeds other forms of support $(\mathcal{R} \ll 1), \rho_{0}=\pi G \Sigma^{2}(1+\chi) /\left(2 \sigma_{z}^{2}\right)$. Substituting this expression for $\rho_{0}$ in Equation (20), the star formation rate can be written as

$$
\begin{aligned}
\Sigma_{\mathrm{SFR}}= & \frac{4 \varepsilon_{\mathrm{ff}}\left(\rho_{0}\right)(1+\chi)^{1 / 2}}{\sqrt{3}} \frac{G \Sigma^{2}}{\sigma_{z}} \\
= & 0.051 M_{\odot} \mathrm{kpc}^{-2} \mathrm{yr}^{-1}(1+\chi)^{1 / 2}\left(\frac{\varepsilon_{\mathrm{ff}}\left(\rho_{0}\right)}{0.005}\right) \\
& \times\left(\frac{\sigma_{z}}{10 \mathrm{~km} \mathrm{~s}^{-1}}\right)^{-1}\left(\frac{\Sigma}{100 M_{\odot} \mathrm{pc}^{-2}}\right)^{2} .
\end{aligned}
$$

Thus, disks dominated by self-gravity rather than external gravity $(\chi \ll 1)$ will have $\Sigma_{\text {SFR }} \propto \Sigma^{2}$ if $\varepsilon_{\mathrm{ff}}\left(\rho_{0}\right) \sim$ constant and the velocity dispersion varies only weakly with $\Sigma$-as we verify below.

We now focus on the case in which turbulence driven by star formation dominates the vertical support and control of star formation. In this limit, equating Equation (13) with Equation (21) for $\sigma_{z} \rightarrow v_{z}$ yields

$$
\begin{aligned}
v_{z} & =\frac{2 f_{P} \varepsilon_{\mathrm{ff}}\left(\rho_{0}\right) p_{*}}{\sqrt{3} \pi m_{*}} \frac{1}{(1+\chi)^{1 / 2}} \\
& =5.5 \mathrm{~km} \mathrm{~s}^{-1} \frac{f_{p}}{(1+\chi)^{1 / 2}}\left(\frac{\varepsilon_{\mathrm{ff}}\left(\rho_{0}\right)}{0.005}\right)\left(\frac{p_{*} / m_{*}}{3000 \mathrm{~km} \mathrm{~s}^{-1}}\right) .
\end{aligned}
$$

The only dependence of the velocity dispersion on the mean gaseous surface density $\Sigma$ is through $\chi$; if the disk is marginally unstable $(Q \lesssim 2)$, however, $\chi \lesssim 0.5$ (see Section 2 ). In the supernova-driven, turbulence-dominated limit, the velocity dispersion is therefore expected to vary only weakly with $\Sigma$ for disks that are marginally gravitationally unstable. The numerical simulations described in Section 3 indeed show a very weak dependence of $v_{z}$ on $\Sigma$, with turbulent velocity dispersions varying by a factor of two $\left(\sim 4-9 \mathrm{~km} \mathrm{~s}^{-1}\right)$ in models with gas surface density between $\Sigma=100 M_{\odot} \mathrm{pc}^{-2}$ and $\Sigma=800 M_{\odot} \mathrm{pc}^{-2}$, which have $\Sigma_{\mathrm{SFR}}$ varying by more than a factor of 100 . The main parameter controlling the velocity dispersion, in disks that cool strongly and therefore have highly supersonic turbulence, is the specific momentum input rate associated with star formation, $p_{*} / m_{*}$. In Equation (22), the fiducial value of $p_{*} / m_{*}$ chosen is that associated with cooled supernova shells. In addition to the direct momentum input from radiative blast waves, cosmic rays that are accelerated by blast waves may drive additional turbulence in escaping from the disk (e.g., via Parker instabilities), which would raise the value of $p_{*}$ and increase $v_{z}$.

By combining Equations (22) and (5) (for $\mathcal{R} \ll 1$ ), we can solve for $\chi$ and $C=\chi(1+\chi)$ in terms of the star formation parameters and the gas disk and bulge properties. Defining

$$
\begin{gathered}
\mathcal{A} \equiv \frac{2 \zeta_{d}}{\pi}\left(\frac{4 f_{P} \varepsilon_{\mathrm{ff}}\left(\rho_{0}\right) p_{*}}{3 \pi m_{*}}\right)^{2} \frac{\rho_{b}}{G \Sigma^{2}} \\
=0.11\left(\frac{\varepsilon_{\mathrm{ff}}\left(\rho_{0}\right)}{0.005}\right)^{2}\left(\frac{p_{*} / m_{*}}{3000 \mathrm{~km} \mathrm{~s}^{-1}}\right)^{2}\left(\frac{\Omega}{0.1 \mathrm{Myr}^{-1}}\right)^{2} \\
\times\left(\frac{\Sigma}{100 M_{\odot} \mathrm{pc}^{-2}}\right)^{-2}
\end{gathered}
$$


we obtain the implicit relation $\chi(1+\chi)^{2}=\mathcal{A}$. An approximate solution good to within $15 \%$ over all $\mathcal{A}$ is

$$
\chi \approx \frac{\mathcal{A}}{1+\mathcal{A}^{2 / 3}} .
$$

For $\mathcal{A} \ll 1, \chi \approx C \approx \mathcal{A}$, and the Toomre parameter is $Q=2.5\left(\sigma_{R} / \sigma_{z}\right) \mathcal{A}^{1 / 2}$.

In the turbulence-dominated limit $\sigma_{z} \approx v_{z}$ (and for $\mathcal{R} \ll 1$ ), the mean midplane density $\rho_{0} \equiv n_{0}\left(1.4 m_{H}\right)$ (for $m_{H}$ the hydrogen mass) and half-thickness $H \equiv \Sigma /\left(2 \rho_{0}\right)$ are given by

$$
\rho_{0}=\frac{(1+\chi) \pi}{2} \frac{G \Sigma^{2}}{v_{z}^{2}}=\frac{3 \pi}{2}(1+\chi)^{2}\left(\frac{\pi m_{*}}{2 f_{p} \varepsilon_{\mathrm{ff}}\left(\rho_{0}\right) p_{*}}\right)^{2} G \Sigma^{2}
$$

so that

$$
\begin{aligned}
n_{0}= & 64 \mathrm{~cm}^{-3} \frac{(1+\chi)^{2}}{f_{p}^{2}}\left(\frac{\varepsilon_{\mathrm{ff}}\left(\rho_{0}\right)}{0.005}\right)^{-2}\left(\frac{p_{*} / m_{*}}{3000 \mathrm{~km} \mathrm{~s}^{-1}}\right)^{-2} \\
& \times\left(\frac{\Sigma}{100 M_{\odot} \mathrm{pc}^{-2}}\right)^{2}
\end{aligned}
$$

and

$$
\begin{aligned}
H= & \frac{1}{(1+\chi)} \frac{v_{z}^{2}}{\pi G \Sigma^{2}}=\frac{1}{3 \pi} \frac{1}{(1+\chi)^{2}}\left(\frac{2 f_{p} \varepsilon_{\mathrm{ff}}\left(\rho_{0}\right) p_{*}}{\pi m_{*}}\right)^{2} \frac{1}{G \Sigma} \\
= & 23 \mathrm{pc} \frac{f_{p}^{2}}{(1+\chi)^{2}}\left(\frac{\varepsilon_{\mathrm{ff}}\left(\rho_{0}\right)}{0.005}\right)^{2}\left(\frac{p_{*} / m_{*}}{3000 \mathrm{~km} \mathrm{~s}^{-1}}\right)^{2} \\
& \times\left(\frac{\Sigma}{100 M_{\odot} \mathrm{pc}^{-2}}\right)^{-1} .
\end{aligned}
$$

We have written Equations (13), (22), (26), and (28) in such a way as to highlight the dependence on the gas surface density. As $\Sigma$ increases, and taking $\mathcal{A}, \chi \ll 1$, the surface density of star formation increases as $\Sigma_{\mathrm{SFR}} \propto \Sigma^{2} m_{*} / p_{*}$, the vertical velocity dispersion is constant $v_{z} \propto \varepsilon_{\mathrm{ff}}\left(\rho_{0}\right) p_{*} / m_{*}$, the mean midplane gas density increases as $\rho_{0} \propto \Sigma^{2}\left(\varepsilon_{\mathrm{ff}}\left(\rho_{0}\right) p_{*} / m_{*}\right)^{-2}$ and the scale height decreases as $H \propto \Sigma^{-1}\left(\varepsilon_{\mathrm{ff}}\left(\rho_{0}\right) p_{*} / m_{*}\right)^{2}$. Increasing the specific momentum input $p_{*} / m_{*}$ from star formation decreases the star formation rate and increases the velocity dispersion and disk thickness. The absence of any explicit timescale in Equation (13) is a signature of self-regulation: the star formation rate must adjust until the turbulence driven by feedback from young stars provides a pressure that matches the vertical weight of the gas. If the turbulence level were lower than the equilibrium value, the mean density would be higher than equilibrium $\left(\rho \propto v_{z}^{-2}\right)$, which would then lead to a shorter dynamical time $t_{\mathrm{ff}} \propto \rho^{-1 / 2}$ and consequently (from Equations (20) and (21)) a higher star formation rate, driving the velocity dispersion upward. Similarly, too high a turbulence level would lead to lower-than-equilibrium $\rho_{0}$ and $\Sigma_{\mathrm{SFR}}$, which would reduce turbulent driving from star formation and eventually lead to a lower value of $v_{z}$.

Although a balance between turbulent driving and dissipation has not been explicitly used in order to derive the above results, it is straightforward to see that these considerations yield equivalent results. The turbulent vertical momentum per unit time per unit gas mass that is driven by stellar inputs is $\sim p_{*} \Sigma_{\mathrm{SFR}} /\left(\Sigma m_{*}\right)$. Since the dissipation time for turbulence is comparable to the flow crossing time over the largest scale (Stone et al. 1998; Mac Low et al. 1998), which in this case is the disk thickness $H \sim v_{z}^{2} /(G \Sigma)$, the dissipation rate of momentum per unit gas mass is $\sim v_{z}^{2} / H \sim G \Sigma$. Equating driving with dissipation yields $\Sigma_{\mathrm{SFR}} \sim G \Sigma^{2} m_{*} / p_{*}$, which is the same as Equation (13) up to order-unity dimensionless constants and the factor $f_{p}$ that has been incorporated to parameterize the details of the momentum injection and mixing.

In terms of the feedback and disk parameters, the Toomre parameter for turbulence-dominated disks is

$$
\begin{aligned}
Q= & 0.8 \frac{1}{(1+\chi)^{1 / 2}} \frac{\sigma_{R}}{\sigma_{z}}\left(\frac{\varepsilon_{\mathrm{ff}}\left(\rho_{0}\right)}{0.005}\right)\left(\frac{p_{*} / m_{*}}{3000 \mathrm{~km} \mathrm{~s}^{-1}}\right) \\
& \times\left(\frac{\Omega}{0.1 \mathrm{Myr}^{-1}}\right)\left(\frac{\Sigma}{100 M_{\odot} \mathrm{pc}^{-2}}\right)^{-1} .
\end{aligned}
$$

Thus, for $\Sigma$ and $\Omega$ in the range observed for galactic center starbursts, the disk will be near the margin of instability. Even if $Q \gtrsim 2$, the intermittency of turbulence implies that some regions may temporarily become unstable. If a large region of the disk becomes unstable and begins to contract, a gravitationally bound supercloud may form.

In a gravitationally bound supercloud, the outward force $F_{\text {kin }}$ from direct kinetic feedback from star formation varies as $\sim \dot{M}_{*} p_{*} / m_{*}$, whereas the outward force $F_{\text {rad }}$ from reprocessed radiation varies as $\sim L_{*} \tau / c \propto \dot{M}_{*} M / r^{2}$, where $\dot{M}_{*}, L_{*}, M$, and $r$ are the supercloud's star formation rate, luminosity, mass, and radius. The inward gravitational force $F_{\text {grav }}$ varies as $\sim G M^{2} / r^{2}$. If $\dot{M}_{*} \propto M / t_{\text {ff }}(\bar{\rho}) \propto r^{-3 / 2}$, then $F_{\text {kin }} / F_{\text {grav }} \propto r^{1 / 2}$ (decreasing as a supercloud shrinks), whereas $F_{\text {rad }} / F_{\text {grav }} \propto r^{-3 / 2}$ (increasing as a supercloud shrinks). This suggests that superclouds, if they become bound, could be destroyed by radiation pressure feedback but not by kinetic feedback. In sufficiently optically thick disks, radiation pressure might prevent supercloud formation; a simple estimate in Appendix B suggests that disks with gas surface densities up to $\Sigma \sim\left(3000 / \kappa_{\mathrm{IR}}\right) M_{\odot} \mathrm{pc}^{-2}$ may be susceptible to supercloud formation.

\section{SUMMARY AND DISCUSSION}

Increasingly high-resolution, multiwavelength observations of galaxies have refined empirical relations between the gas content and mean star formation rate in galaxies. The picture now emerging appears to include at least three regimes: outerdisks, mid-disks, and galactic centers. The first and last regimes evidence superlinear mean relations between the surface density of star formation $\Sigma_{\mathrm{SFR}}$ and the gas surface density $\Sigma$, while the relation is close to linear for mid-disks.

From a theoretical point of view, star formation in all regimes is likely to be self-regulated in some way. For the outerdisk regime, Ostriker et al. (2010) have proposed that UV feedback from massive young stars is particularly important to self-regulation. In this model, the star formation rate in outer disks must be such that the heating of diffuse atomic gas by stellar UV radiation balances cooling, with the cooling rate set by the gas pressure (and hence responsive to the vertical gravity of the disk). For the mid-disk regime, gas resides predominantly in gravitationally bound (but transient) GMCs with mean densities (and hence star formation rates) that depend on the amplitude of supersonic turbulence driven by star formation feedback (possibly by expanding $\mathrm{H}$ II regions, although this is not well understood). For galactic center regions, we argue in this paper that the momentum injected to the disk by massive star formation is crucial to establishing a self-regulated state. Vertical equilibrium of the disk requires gravity to be 
balanced by an outward momentum flux, consisting primarily of turbulent pressure (largely driven by supernovae) for most molecule-dominated central regions. Our analysis suggests that the galactic-center star formation regime may further subdivide, with a transition to radiation-dominated momentum flux (or pressure) only for extremely high $\Sigma$, highly opaque disks.

The analysis of Section 2 provides a prediction (see Equation (10)) for the dependence of $\Sigma_{\mathrm{SFR}}$ on $\Sigma$ and the density $\rho_{b}$ of the stellar bulge, parameterized by the mean specific momentum $p_{*} / m_{*}$ injected by star formation to the ISM (primarily from supernovae), by the mean IR opacity $\kappa_{\mathrm{IR}}$ (which determines how much reprocessed starlight is trapped), and by the mass-to-radiation energy efficiency of star formation $\epsilon_{*}$ (which depends on the IMF). Assuming that gas gravity dominates the vertical potential, self-regulated, turbulencedominated disks are expected to have $\Sigma_{\mathrm{SFR}} \approx 2 \pi m_{*} G \Sigma^{2} / p_{*}$ (see Equation (13)), whereas radiation-dominated disks are expected to have $\Sigma_{\mathrm{SFR}} \approx 2 \pi G \Sigma /\left(\epsilon_{*} c \kappa_{\mathrm{IR}}\right)$ (see Equation (14)). The turbulence-dominated regime is expected to apply for $\Sigma \sim 100-10^{4} M_{\odot} \mathrm{pc}^{-2}$, covering most starbursts (although not the innermost disk regions that merge into active galactic nucleus disks). For the radiation-dominated limit, an equivalent expression to our result was previously obtained (under somewhat different assumptions) by Thompson et al. (2005, see their Equation (28)), and they also noted that optically thin disks are expected to have a steeper dependence of $\Sigma_{\mathrm{SFR}}$ on $\Sigma$ than optically thick disks.

In a self-regulated disk, the feedback parameters determine the star formation rate, independent of the internal structure of the disk. Thus, the vertically integrated star formation rates of Equations (13) and (14) depend only on the vertically integrated disk properties. However, it is natural that the star formation rate should also connect to the timescale for gas to become concentrated into high-density clouds, as discussed in Section 5. For a system in equilibrium where the gas is confined primarily by its own gravitational potential, this timescale is the gravitational free-fall time at the mean density; for a disk, $1 / t_{\mathrm{ff}}(\bar{\rho}) \propto(G \Sigma / H)^{1 / 2}$. The mean density (or disk thickness), in turn, depends on what is balancing gravity - thermal pressure, turbulent pressure, or radiation pressure. In the turbulencedominated, gas-gravity dominated case, $H=v_{z}^{2} /(\pi G \Sigma)$ for $v_{z}$ the vertical velocity dispersion, which yields $1 / t_{\mathrm{ff}}(\bar{\rho})=$ $4 G \Sigma /\left(\sqrt{3} v_{z}\right)$. If $\varepsilon_{\mathrm{ff}}\left(\rho_{0}\right)$ is the collapse efficiency per free-fall time at the mean midplane density, the star formation rate is given by $\Sigma_{\mathrm{SFR}}=2.3 \varepsilon_{\mathrm{ff}}\left(\rho_{0}\right) G \Sigma^{2} / v_{z}$ (see Equation (21)).

Krumholz \& McKee (2005) proposed that the lognormal density distribution in highly turbulent systems will lead to a star-forming efficiency declining as the $-1 / 3$ power of the turbulent Mach number. Including this scaling for $\varepsilon_{\mathrm{ff}}\left(\rho_{0}\right)$ would lead to $\Sigma_{\mathrm{SFR}} \propto \Sigma^{2} v_{z}^{-1.3}$. Under the assumption that in moleculedominated gas $\Sigma_{\mathrm{SFR}}=\varepsilon_{\mathrm{ff}}(\rho) \Sigma / t_{\mathrm{ff}}(\rho)$, Krumholz et al. (2009) proposed a power-law relation between $\Sigma_{\text {SFR }}$ and $\Sigma$ in galactic center regions. In obtaining this relation, they assumed that the Toomre parameter $Q \approx 1$, and also that $\Omega \propto \Sigma^{0.5}$; together these would imply that velocity dispersion varies $\propto \Sigma^{0.5}$. Although we in fact argue that the vertical velocity dispersion $v_{z}$ is rather insensitive to $\Sigma$, inserting $v_{z} \propto \Sigma^{0.5}$ in $\Sigma_{\mathrm{SFR}} \propto \Sigma^{2} v_{z}^{-1.3}$ would yield $\Sigma_{\mathrm{SFR}} \propto \Sigma^{1.3}$, the result given in Equation (10) of Krumholz et al. (2009).

In order for the disk to form stars at a rate controlled by the free-fall time $\left(\Sigma_{\mathrm{SFR}} \approx 2.3 \varepsilon_{\mathrm{ff}}\left(\rho_{0}\right) G \Sigma^{2} / v_{z}\right)$, and also for the momentum feedback from star formation to control vertical equilibrium of the disk $\left(\Sigma_{\mathrm{SFR}} \approx 2 \pi m_{*} G \Sigma^{2} / p_{*}\right)$, the vertical velocity dispersion must adjust to $v_{z} \approx 0.4 \varepsilon_{\mathrm{ff}}\left(\rho_{0}\right) p_{*} / m_{*}$ (see Equation (22)). Interestingly, this is independent of the disk surface density and the star formation rate; our numerical simulations in fact show that $v_{z}$ is insensitive to the star formation rate. Other recent numerical simulations have also indicated that turbulent velocity dispersions vary relatively weakly with the input star formation rate (Dib et al. 2006; Shetty \& Ostriker 2008; Agertz et al. 2009; Joung et al. 2009). The relation $v_{z} \sim \varepsilon_{\mathrm{ff}}\left(\rho_{0}\right) p_{*} / m_{*}$ can also be understood as simply a balance between driving turbulent velocities in the ISM at a rate $\sim p_{*} \varepsilon_{\mathrm{ff}}\left(\rho_{0}\right) /\left[m_{*} t_{\mathrm{ff}}\left(\rho_{0}\right)\right]$, and dissipating turbulent velocities at a rate $\sim v_{z} v_{z} / H \sim v_{z} / t_{\mathrm{ff}}\left(\rho_{0}\right)$. For $\varepsilon_{\mathrm{ff}}\left(\rho_{0}\right) \sim 0.01$ and $p_{*} / m_{*} \sim 3000 \mathrm{~km} \mathrm{~s}^{-1}$, the velocity dispersion driven by star formation feedback is $v_{z} \sim 10 \mathrm{~km} \mathrm{~s}^{-1}$.

More generally, if the vertical momentum injection rate per unit ISM gas mass from star formation is $\sim(1-2) p_{*} /$ $\left(4 m_{*} t_{\mathrm{SF}, \mathrm{gas}}\right)$, and the corresponding turbulent dissipation rate is $\sim v_{z} /\left[(1-2) t_{\mathrm{dyn}}\right]$ for vertical dynamical time $t_{\mathrm{dyn}}=H / \sigma_{z}$, the expected vertical velocity dispersion is $v_{z} \sim(0.3-1)$ $\left(p_{*} / m_{*}\right) t_{\mathrm{dyn}} / t_{\mathrm{SF}, \mathrm{gas}}$. This is within a factor of a few of $10 \mathrm{~km} \mathrm{~s}^{-1}$ if $p_{*} / m_{*} \sim 3000 \mathrm{~km} \mathrm{~s}^{-1}$ from supernovae and $t_{\mathrm{SF} \text {, gas }} \sim 100 t_{\mathrm{dyn}}$ (which is true in outer disks as well as starbursts; see below), regardless of whether the vertical dynamical time is set by gas self-gravity or by gravity of an external potential (stellar bulge, stellar disk, or dark matter halo). Velocity dispersions reported for starburst regions are often much larger than the values we find (e.g., Downes \& Solomon 1998; Genzel et al. 2010b), but it is difficult observationally to eliminate sub-beam sheared rotation, radial/azimuthal streaming, and other non-turbulent (and nonvertical) motions on the relevant scales $(\sim H \ll 100$ pc). Observations of molecular velocity dispersions within the central $\mathrm{kpc}$ of a few nearby, face-on galaxies are $\sim 10 \mathrm{~km} \mathrm{~s}^{-1}$ (Combes \& Becquaert 1997); turbulent molecular velocity dispersions from larger scales are also similar (Wilson et al. 2010).

The momentum-regulated, turbulence-dominated prediction of Equation (13) for $\Sigma_{\mathrm{SFR}}$ can be compared to the results of numerical simulations and to observations. We do this in Sections 3 and 4, respectively. The simulations indeed yield a relation consistent with the prediction, $\Sigma_{\mathrm{SFR}} \approx$ $0.1 M_{\odot} \mathrm{kpc}^{-2} \mathrm{yr}^{-1}\left(\Sigma / 100 M_{\odot} \mathrm{pc}^{-2}\right)^{2}$, adopting a value of the momentum input per unit stellar mass formed of $p_{*} / m_{*}=$ $3000 \mathrm{~km} \mathrm{~s}^{-1}$. Velocity dispersions from the simulations are also comparable to prediction. Further comparisons of the star formation rates and disk properties from the model with the results of numerical simulations will be presented by R. Shetty \& E. C. Ostriker (2011, in preparation).

A difficulty in comparing to observations is that the molecular mass must be obtained indirectly through observations of $\mathrm{CO}$ transitions. Although the conversion factor $X_{\mathrm{CO}}$ from integrated $\mathrm{CO}$ intensity to hydrogen column density has been well calibrated empirically using several different methods for individual GMCs and main-disk regions which integrate over collections of GMCs, the value of $X_{\mathrm{CO}}$ is much less certain in galactic center regions. There is evidence that $X_{\mathrm{CO}}$ decreases in high- $\Sigma$ starburst regions; this may owe to a combination of factors, including higher $\mathrm{CO}$ excitation in the warmer, denser gas, a larger fraction of the gas that is sufficiently shielded to create $\mathrm{CO}$, and larger velocity gradients that allow radiation to escape more easily. We compare to the large sample of observations compiled in Genzel et al. (2010a). If the conversion factor is assumed to decrease with increasing integrated $\mathrm{CO}$ intensity (as $X_{\mathrm{CO}} \propto I_{\mathrm{CO}}^{-0.3}$ ), the observed and predicted relations for $\Sigma_{\mathrm{SFR}}$ versus $\Sigma$ are in excellent agreement, for the range $\Sigma \sim 10^{2}-10^{4} M_{\odot} \mathrm{pc}^{-2}$. If two 
different constant conversion factors are adopted for normal and merger systems, the former agree with the predicted $\Sigma_{\mathrm{SFR}}$ at low $\Sigma$, and the latter agree at high $\Sigma$. To make progress in relating theory to observations, a top priority is to determine how $X_{\mathrm{CO}}$ varies under the conditions that prevail in galactic center regions. This will require both empirical calibrations, and radiative transfer models applied to numerical simulations that resolve the structure of turbulent disks.

Finally, we note that the star formation timescale $t_{\mathrm{SF}, \mathrm{gas}}=$ $\Sigma / \Sigma_{\mathrm{SFR}} \approx p_{*} /\left(2 \pi G m_{*} \Sigma\right)$ in self-regulated, turbulencedominated, molecular galactic center disks can also be expressed as $t_{\mathrm{SF}, \text { gas }} \sim t_{\mathrm{dyn}} / \varepsilon_{\mathrm{ff}}\left(\rho_{0}\right) \sim 100 t_{\mathrm{dyn}}$, for vertical dynamical time $t_{\text {dyn }}=H / \sigma_{z}$. In Ostriker et al. (2010), we showed ${ }^{6}$ that in outer disks, self-regulation via UV heating of atomic gas leads to a quantitatively similar result, $t_{\mathrm{SF}, \mathrm{gas}} \sim 200 \mathrm{H} / \sigma_{z}$. The constant factor $\sim 200$ in the outer-disk formula for $t_{\mathrm{SF} \text {, gas }}$ is set, however, by $\sigma_{z}$ and the ratio of heating and cooling rate coefficients for atomic gas and by the UV production efficiency of the youngstar population, rather than just by the turbulent processes that set the constant factor $\sim 1 / \varepsilon_{\mathrm{ff}}\left(\rho_{0}\right)$ in the galactic-center formula. Thus, although different physical processes control star formation in galactic centers and outer disks, the dependence of $\Sigma_{\mathrm{SFR}}$ on $1 / t_{\mathrm{dyn}}$ (and on $\Omega \sim Q / t_{\mathrm{dyn}}$ ) is similar, both in terms of the scaling and the coefficient. We believe that this dependence on $1 / t_{\mathrm{dyn}}$, together with the limited range of $Q$ that disk evolution yields, is the reason that both whole galaxies and central starbursts, at a range of redshifts, tend to follow the empirical relation $\Sigma_{\mathrm{SFR}} \sim 0.02 \Sigma \Omega$ (Kennicutt 1998b; Daddi et al. 2010; Genzel et al. 2010a).

We are grateful to Reinhard Genzel for providing a table based on his compilation of galactic gas content and star formation rates. We thank the referee for helpful suggestions on the manuscript. The work of E.C.O. was supported by a fellowship from the John Simon Guggenheim Foundation and by grant AST-0908185 from the National Science Foundation. R.S. acknowledges support from the German Bundesministerium für Bildung und Forschung via the ASTRONET project STAR FORMAT (grant 05A09VHA).

\section{APPENDIX A}

\section{MOMENTUM INJECTION TO THE ISM FROM RADIATION-DRIVEN SHELLS AROUND STAR CLUSTERS}

Consider a GMC of mass $M_{\mathrm{GMC}}$ which forms stars with a net efficiency over its lifetime of $\varepsilon_{\mathrm{GMC}} \equiv M_{*} / M_{\mathrm{GMC}}$, ejecting the remaining gas mass $\left(1-\varepsilon_{\mathrm{GMC}}\right) M_{\mathrm{GMC}}$. Treating the stellar component as collected in a single central cluster of (fixed) radius $r_{\mathrm{cl}}$ and luminosity $L_{*}=\varepsilon_{\mathrm{GMC}} \Psi M_{\mathrm{GMC}}$, and the ejected mass as an expanding spherical shell of (variable) radius $r$ surrounding it, the total radiation force applied to the interior of the shell by reprocessed (diffuse) radiation is $F_{\text {rad }}=4 \pi r^{2}\left(a_{\text {rad }} / 3\right) T_{\text {int }}^{4}$, where $T_{\text {int }}^{4}=(3 / 4) \tau T_{\text {eff }}^{4}$ for shell optical depth $\tau \gg 1$ and internal and surface (effective) temperatures $T_{\text {int }}$ and $T_{\text {eff. Equating }} L_{*}$ with $4 \pi r^{2}\left(a_{\mathrm{rad}} c / 4\right) T_{\mathrm{eff}}^{4}$ and using $\tau=\kappa_{\mathrm{IR}}\left(1-\varepsilon_{\mathrm{GMC}}\right) M_{\mathrm{GMC}} /\left(4 \pi r^{2}\right), F_{\mathrm{rad}}=L_{*} \tau / c=$ $\kappa_{\mathrm{IR}}\left(1-\varepsilon_{\mathrm{GMC}}\right) \varepsilon_{\mathrm{GMC}} \Psi M_{\mathrm{GMC}}^{2} /\left(4 \pi c r^{2}\right)$. The gravitational force on the shell, including that of the central cluster and its own selfgravity, is $-G M_{\mathrm{GMC}}^{2}\left(1-\varepsilon_{\mathrm{GMC}}^{2}\right) /\left(2 r^{2}\right)$. The net acceleration of

\footnotetext{
6 See Equation (A16) in Ostriker et al. (2010), note that $t_{\text {con }}$ there is the same
} as $t_{\mathrm{SF}, \text { gas }}$ here. the shell is therefore

$$
\begin{aligned}
\ddot{r} & =\frac{G M_{\mathrm{GMC}}}{r^{2}}\left[\frac{\kappa_{\mathrm{IR}} \Psi \varepsilon_{\mathrm{GMC}}}{4 \pi G c}-\frac{\left(1+\varepsilon_{\mathrm{GMC}}\right)}{2}\right] \\
& =\frac{\left(\psi \varepsilon_{\mathrm{GMC}}-1\right)}{2} \frac{G M_{\mathrm{GMC}}}{r^{2}}
\end{aligned}
$$

for $\psi \equiv \Psi \kappa_{\mathrm{IR}} /(2 \pi c G)-1$. As pointed out by Murray et al. (2010), this implies that there is a minimum value of $\varepsilon_{\mathrm{GMC}}$ for the pressure associated with reprocessed radiation to disrupt the cloud; the shell can only become unbound if $\varepsilon_{\mathrm{GMC}}>\psi^{-1} \equiv$ $\varepsilon_{\min }$.

Equation (A1) can be integrated to obtain the asymptotic speed $v_{f}=\left[\left(\psi \varepsilon_{\mathrm{GMC}}-1\right) G M_{\mathrm{GMC}} / r_{\mathrm{cl}}\right]^{1 / 2}$ of the shell, assuming its initial radius is $r_{\mathrm{cl}}$. The resulting ratio of the momentum in the shell divided by the total mass in stars formed, representing $p_{*} / m_{*}$ for a radiation-driven shell, is $v_{f}\left(1-\varepsilon_{\mathrm{GMC}}\right) / \varepsilon_{\mathrm{GMC}}$. For any given value of $\psi$, it is straightforward to show that the maximum momentum-to-mass ratio is obtained when $\varepsilon_{\mathrm{GMC}}=4 \varepsilon_{\min }\left[1+\left(1+8 \varepsilon_{\min }\right)^{1 / 2}\right]^{-1} \equiv$ $\varepsilon_{\text {max }}$. Using $\Psi=2000 \mathrm{erg} \mathrm{s}^{-1} \mathrm{~g}^{-1}$ (Dopita et al. 2006) for young, luminous clusters that fully sample the Kroupa (2001) IMF and $\kappa_{\mathrm{IR}}=20 \mathrm{~cm}^{2} \mathrm{~g}^{-1}$ for warm, dusty shells (Murray et al. 2010; Semenov et al. 2003), this yields $\varepsilon_{\min }=$ $0.5, \varepsilon_{\max }=0.6$, and $\left[p_{*} / m_{*}\right]_{\mathrm{rad}}=0.4\left(G M_{\mathrm{GMC}} / r_{\mathrm{cl}}\right)^{1 / 2}=$ $30 \mathrm{~km} \mathrm{~s}^{-1}\left(M_{\mathrm{GMC}} / 10^{6} M_{\odot}\right)^{1 / 2}\left(r_{\mathrm{cl}} / \mathrm{pc}\right)^{-1 / 2}$. This is comparable to the velocity dispersion of the cluster itself, which even for the most massive clusters in starbursts does not exceed $100 \mathrm{~km} \mathrm{~s}^{-1}$ and is typically much lower (McCrady et al. 2003; Maraston et al. 2004; Overzier et al. 2009).

In addition to diffuse (reprocessed) radiation, streaming radiation (dominated by UV) from the star cluster also imparts momentum to the expanding gas cloud (or shell) where it is first absorbed. The maximum force from this streaming radiation is $L_{*} / c$, such that the maximum contribution to $v_{f}\left(1-\varepsilon_{\mathrm{GMC}}\right) / \varepsilon_{\mathrm{GMC}}=p_{*} / m_{*}$ is $\int \Psi d t / c=\epsilon_{*} c \sim 200 \mathrm{~km} \mathrm{~s}^{-1}$. Thus, allowing for both reprocessed and direct stellar radiation, the value of $p_{*} / m_{*}$ from radiation-driven expanding shells is smaller than the momentum-per-stellar mass injected to the disk by radiative supernovae shells, $\sim 3000 \mathrm{~km} \mathrm{~s}^{-1}$.

\section{APPENDIX B}

\section{SUPERCLOUD EVOLUTION}

Potentially, large-scale instabilities in the disk can lead to gaseous supercloud formation. How would a supercloud evolve if it is able to form? For a spherical, uniform-density cloud of mass $M$ and radius $r$ that forms stars at a rate $\dot{M}_{*}=$ $\varepsilon_{\mathrm{ff}}(\bar{\rho}) M / t_{\mathrm{ff}}(\bar{\rho})$, producing a luminosity $L_{*}=\epsilon_{*} c^{2} \dot{M}_{*}$ and a supernova rate $\dot{M}_{*} / m_{*}$, the ratio of the outward force (due to the combined momentum input from supernovae and reprocessed radiation pressure ${ }^{7}$ ) to the inward force (due to gravity) is

$$
\frac{F_{+}}{F_{-}}=\frac{2^{7 / 2} \varepsilon_{\mathrm{ff}}(\bar{\rho})}{3 \pi G^{1 / 2} M^{1 / 2}}\left[f_{p} \frac{p_{*}}{m_{*}} r^{1 / 2}+\frac{3 \epsilon_{*} c \kappa_{\mathrm{IR}} M}{4 \pi} r^{-3 / 2}\right] \text {. }
$$

The contribution to $F_{+} / F_{-}$from supernovae declines $\propto r^{1 / 2}$ as $r$ decreases, whereas the contribution to $F_{+} / F_{-}$from radiation increases $\propto r^{-3 / 2}$. Thus, radiation forces become increasingly

\footnotetext{
7 Streaming radiation adds a term $<\epsilon_{*} c \sim 200 \mathrm{~km} \mathrm{~s}^{-1}$ to $p_{*} / m_{*}$ that is small compared to the contribution $p_{*} / m_{*} \approx 3000 \mathrm{~km} \mathrm{~s}^{-1}$ from supernovae, and thus may be neglected.
} 
important as the cloud shrinks; eventually, if $F_{+} / F_{-}$becomes $>1$, inward contraction will be halted. If the star formation efficiency is sufficient, the cloud will ultimately be destroyed, with a substantial fraction of its mass once again becoming unbound (cf. Krumholz \& Matzner 2009; Murray et al. 2010; Krumholz \& Dekel 2010; and the discussion in Appendix A).

The masses of clouds that form via gravitational instability in disks with $Q<Q_{\text {crit }}$ are typically $\sim 1-10 M_{\text {J,2D }}$ (e.g., Kim et al. 2002, 2003; Kim \& Ostriker 2007), where $M_{\mathrm{J}, 2 \mathrm{D}}=$ $(\pi H)^{2} \Sigma=\sigma^{4} /\left(G^{2} \Sigma\right)$ for $\sigma$ the gas velocity dispersion. Letting $M \equiv m M_{\mathrm{J}, 2 \mathrm{D}}, r \equiv x H$, and assuming $\sigma=v_{z}$, we can use Equations (22), (28), (11), and (12) to obtain

$$
\frac{F_{+}}{F_{-}}=\frac{1.8}{m^{1 / 2}}\left[x^{1 / 2}+\frac{3 \pi}{4} \frac{\tau}{\tau_{*}} \frac{m}{x^{3 / 2}}\right],
$$

where $\tau$ is evaluated using the unperturbed disk surface density $\Sigma$, and we have assumed $\chi \ll 1$.

For a given $m$, the force ratio has a minimum at $x_{\min }=2.7\left(m \tau / \tau_{*}\right)^{1 / 2}$, where its value is $\left(F_{+} / F_{-}\right)_{\min }=$ $4.0 m^{-1 / 4}\left(\tau / \tau_{*}\right)^{1 / 4}$. Taken at face value, this suggests that only disks with a sufficiently low surface density $\Sigma$ will form contracting superclouds, since only if $\tau / \tau_{*}$ is sufficiently small can $F_{+} / F_{-}$be $<1$. With $m=10, F_{+} / F_{-}<1$ for $\tau / \tau_{*}<0.04$, corresponding to $\Sigma<\left(3000 / \kappa_{\mathrm{IR}}\right) M_{\odot} \mathrm{pc}^{-2}$. Note also that $x_{\min }<1$ only for $\Sigma<\left(1000 / \kappa_{\mathrm{IR}}\right) M_{\odot} \mathrm{pc}^{-2}$. Of course, given the highly idealized assumptions adopted, the particular value obtained from this simple estimate should not be taken too seriously. Physically, however, it is reasonable to expect higher- $\Sigma$ disks that are increasingly radiation-pressure supported to be less subject to gravitational instability, because of their stiff equation of state: if $P_{\text {rad }} \propto L_{*} \rho / r$ and $L_{*} \propto M \rho^{1 / 2}$, the internal radiation pressure in a supercloud would vary as $P \propto \rho^{11 / 6}$. Potentially, more detailed understanding of large-scale gravitational instabilities in starburst disks could be obtained via an analysis similar to that of Thompson (2008), but including sources of radiation and turbulence.

\section{REFERENCES}

Agertz, O., Lake, G., Teyssier, R., Moore, B., Mayer, L., \& Romeo, A. B. 2009, MNRAS, 392, 294

Bigiel, F., Leroy, A., Walter, F., Brinks, E., de Blok, W. J. G., Madore, B., \& Thornley, M. D. 2008, AJ, 136, 2846

Blanc, G. A., Heiderman, A., Gebhardt, K., Evans, N. J., \& Adams, J. 2009, ApJ, 704,842

Blitz, L., Fukui, Y., Kawamura, A., Leroy, A., Mizuno, N., \& Rosolowsky, E. 2007, in Protostars and Planets V, ed. B. Reipurth, D. Jewitt, \& K. Keil (Tucson, AZ: Univ. Arizona Press), 81

Blitz, L., \& Rosolowsky, E. 2004, ApJ, 612, L29

Blitz, L., \& Rosolowsky, E. 2006, ApJ, 650, 933

Bolatto, A. D., Leroy, A. K., Rosolowsky, E., Walter, F., \& Blitz, L. 2008, ApJ, 686, 948

Bothwell, M. S., et al. 2010, MNRAS, 405, 219

Boulares, A., \& Cox, D. P. 1990, ApJ, 365, 544

Chevalier, R. A. 1974, ApJ, 188, 501

Cioffi, D. F., McKee, C. F., \& Bertschinger, E. 1988, ApJ, 334, 252

Combes, F., \& Becquaert, J. 1997, A\&A, 326, 554

Daddi, E., et al. 2010, ApJ, 714, L118

Dame, T. M., Hartmann, D., \& Thaddeus, P. 2001, ApJ, 547, 792

Dib, S., Bell, E., \& Burkert, A. 2006, ApJ, 638, 797

Dopita, M. A., et al. 2006, ApJ, 647, 244

Downes, D., \& Solomon, P. M. 1998, ApJ, 507, 615

Downes, D., Solomon, P. M., \& Radford, S. J. E. 1993, ApJ, 414, L13

Evans, N. J., et al. 2009, ApJS, 181, 321

Ferrière, K. M. 2001, Rev. Mod. Phys., 73, 1031

Gao, Y., \& Solomon, P. M. 2004, ApJ, 606, 271

Gardiner, T. A., \& Stone, J. M. 2005, J. Comput. Phys., 205, 509

Gardiner, T. A., \& Stone, J. M. 2008, J. Comput. Phys., 227, 4123
Genzel, R., et al. 1998, ApJ, 498, 579

Genzel, R., et al. 2010a, MNRAS, 407, 2091

Genzel, R., et al. 2010b, arXiv:1011.5360

Heesen, V., Krause, M., Beck, R., \& Dettmar, R. 2009, A\&A, 506, 1123

Heiderman, A., Evans, N. J., II, Allen, L. E., Huard, T., \& Heyer, M. 2010, ApJ, 723, 1019

Heyer, M., Krawczyk, C., Duval, J., \& Jackson, J. M. 2009, ApJ, 699, 1092

Joung, M. R., Mac Low, M., \& Bryan, G. L. 2009, ApJ, 704, 137

Kennicutt, R. C., Jr. 1998a, ARA\&A, 36, 189

Kennicutt, R. C., Jr. 1998b, ApJ, 498, 541

Kim, C., Kim, W., \& Ostriker, E. C. 2006, ApJ, 649, L13

Kim, W., \& Ostriker, E. C. 2007, ApJ, 660, 1232

Kim, W., Ostriker, E. C., \& Stone, J. M. 2002, ApJ, 581, 1080

Kim, W., Ostriker, E. C., \& Stone, J. M. 2003, ApJ, 599, 1157

Koyama, H., \& Ostriker, E. C. 2009a, ApJ, 693, 1316

Koyama, H., \& Ostriker, E. C. 2009b, ApJ, 693, 1346

Kroupa, P. 2001, MNRAS, 322, 231

Krumholz, M. R., \& Dekel, A. 2010, MNRAS, 406, 112

Krumholz, M. R., \& Matzner, C. D. 2009, ApJ, 703, 1352

Krumholz, M. R., Matzner, C. D., \& McKee, C. F. 2006, ApJ, 653, 361

Krumholz, M. R., \& McKee, C. F. 2005, ApJ, 630, 250

Krumholz, M. R., McKee, C. F., \& Tumlinson, J. 2009, ApJ, 699, 850

Krumholz, M. R., \& Tan, J. C. 2007, ApJ, 654, 304

Lada, C. J., Lombardi, M., \& Alves, J. F. 2010, ApJ, 724, 687

Leitherer, C., et al. 1999, ApJS, 123, 3

Leroy, A. K., Walter, F., Brinks, E., Bigiel, F., de Blok, W. J. G., Madore, B., \& Thornley, M. D. 2008, AJ, 136, 2782

Mac Low, M., \& Klessen, R. S. 2004, Rev. Mod. Phys., 76, 125

Mac Low, M., Klessen, R. S., Burkert, A., \& Smith, M. D. 1998, Phys. Rev. Lett., 80, 2754

Maraston, C., Bastian, N., Saglia, R. P., Kissler-Patig, M., Schweizer, F., \& Goudfrooij, P. 2004, A\&A, 416, 467

Matzner, C. D. 2002, ApJ, 566, 302

McCrady, N., Gilbert, A. M., \& Graham, J. R. 2003, ApJ, 596, 240

McKee, C. F., \& Ostriker, E. C. 2007, ARA\&A, 45, 565

Murray, N., Quataert, E., \& Thompson, T. A. 2010, ApJ, 709, 191

Norman, C. A., \& Ferrara, A. 1996, ApJ, 467, 280

Ostriker, E. C., McKee, C. F., \& Leroy, A. K. 2010, ApJ, 721, 975

Overzier, R. A., et al. 2009, ApJ, 706, 203

Padoan, P., \& Nordlund, A. 2009, arXiv:0907.0248

Piontek, R. A., \& Ostriker, E. C. 2007, ApJ, 663, 183

Reynolds, S. P. 2008, ARA\&A, 46, 89

Rosas-Guevara, Y., Vazquez-Semadeni, E., Gomez, G. C., \& Katharina Jappsen, A. 2010, MNRAS, 406, 1875

Sanders, D. B., \& Mirabel, I. F. 1996, ARA\&A, 34, 749

Scoville, N. Z., Sargent, A. I., Sanders, D. B., \& Soifer, B. T. 1991, ApJ, 366, L5

Semenov, D., Henning, T., Helling, C., Ilgner, M., \& Sedlmayr, E. 2003, A\&A, 410,611

Sheth, K., Vogel, S. N., Wilson, C. D., \& Dame, T. M. 2008, ApJ, 675, 330

Shetty, R., Glover, S. C., Dullemond, C. P., \& Klessen, R. S. 2011, MNRAS, 11

Shetty, R., \& Ostriker, E. C. 2008, ApJ, 684, 978

Solomon, P. M., Downes, D., Radford, S. J. E., \& Barrett, J. W. 1997, ApJ, 478, 144

Solomon, P. M., Rivolo, A. R., Barrett, J., \& Yahil, A. 1987, ApJ, 319, 730

Solomon, P. M., \& Vanden Bout, P. A. 2005, ARA\&A, 43, 677

Spitzer, L. 1978, Physical Processes in the Interstellar Medium (New York: Wiley-Interscience)

Stone, J. M., Gardiner, T. A., Teuben, P., Hawley, J. F., \& Simon, J. B. 2008, ApJS, 178, 137

Stone, J. M., Ostriker, E. C., \& Gammie, C. F. 1998, ApJ, 508, L99

Strong, A. W., \& Mattox, J. R. 1996, A\&A, 308, L21

Tacconi, L. J., et al. 2008, ApJ, 680, 246

Thompson, T. A. 2008, ApJ, 684, 212

Thompson, T. A., Quataert, E., \& Murray, N. 2005, ApJ, 630, 167

Toomre, A. 1964, ApJ, 139, 1217

Tüllmann, R., Dettmar, R., Soida, M., Urbanik, M., \& Rossa, J. 2000, A\&A, 364, L36

Vázquez-Semadeni, E., Gómez, G. C., Jappsen, A., Ballesteros-Paredes, J., \& Klessen, R. S. 2009, ApJ, 707, 1023

Wilson, C. D., et al. 2011, MNRAS, 410, 1409

Wong, T., \& Blitz, L. 2002, ApJ, 569, 157

Wu, J., Evans, N. J., II, Gao, Y., Solomon, P. M., Shirley, Y. L., \& Vanden Bout, P. A. 2005, ApJ, 635, L173

Wu, J., Evans, N. J., Shirley, Y. L., \& Knez, C. 2010, ApJS, 188, 313

Yan, H., \& Lazarian, A. 2008, ApJ, 673, 942

Yusef-Zadeh, F., et al. 2009, ApJ, 702, 178 